\title{
SES2D User's Manual
}

\author{
James D. Johnson \\ Stanford P. Lyon
}

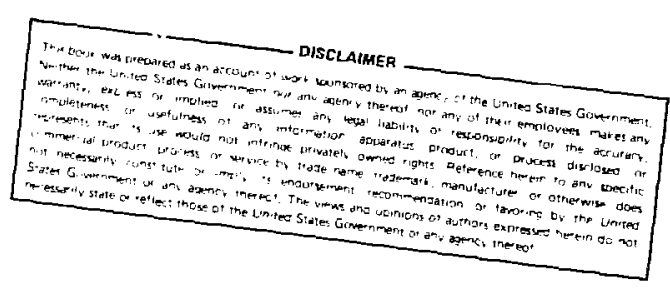




\title{
SES2D User's Manual
}

by

\author{
James D. Johnson and Stanford P. Lyon
}

\begin{abstract}
SES2D is an interactive graphics code designed to generate plots of equation of state data from the Los Alamos National Laboratory Group T-4 computer libraries. This manual discusses the capabilities of the code. It describes the prompts and commands and illustrates their use with a sample run.
\end{abstract}

\section{GENERAL OVERVIEW}

SES2D is an interactive graphics code designed to generate plots of equation of state data. Versions of SES2D for use with sequential and random libraries exist on both the 7600 and Cray computers. In response to code prompts, you may specify a library name, material number, output device, and various parameters associated with the format and type of graph. The default library is SESAME for the open partition and SESAMEA for the closed partition. The output devices are microfilm, microfiche, and the Tektronix terminal. The code will continue to prompt you for more input until the output device parameter is entered. In addition to the type of curve, you may specify linear or logarithmic axes either with or without grid lines. Here the initial default is to $\log -\log$ axes with no grid lines. Once a curve or family of curves has been plotted, you may then modify : e existing plot (for example, by deleting a specific curve or curves from a family of curves). Furthermore, curves may be added to the same grid to compare two or more materials.

Currently, there are four classes of graphs: isotherms, isochores, Hugoniots, and adiabats. To select a particular graph, type a command of the form $y(x) z$, where $z=t$ for isotherms, $z=r$ for isochores, $z=h$ for Hugoniots, and $z=s$ for adiabats. The variable $x$ is plotted on the abscissa and the variable $y$ is plotted on the ordinate. The initial default is to plot pressure isotherms $p(r h o) t$; subsequent defaults are to the last type of graph plotted. What is allowed for $\mathrm{x}$ and $\mathrm{y}$ depends on which class of graph is being plotted. The variables allowed for each class of graph are listed below. Any pair of values may be used for $x$ and $y$.

ISOTHERMS $p$ e rho pdt pdr edt edr eta $v$ ISOCHORES $\quad p$ e $t$ pdt pdr edt edr eta $v$ HUGONIOTS $p$ rho $t$ eta $v$ us up

ADIABATS $p$ tho $t$ eta $v$ up $c$

Here $p$ is pressure; $e$ is energy; rho is density, $t$ is temperature; pdt is the temperature derivative of $p$; $p d r$ is the density derivative of $p$; edt is the temperature derivative of $e$; edr is the density derivative of $e$; eta is rho/rho0, where tho0 is the reference density given in the SESAME table; $v$ is $1 /$ rho; us is the shock 
velocity; up is the particle velocity; and $c$ is the sound velocity. In plotting a curve, $v$ is set equal to $1.0 \mathrm{e} 100$ if tho is less than or equal to zero.

Specific upper and lower limits can be set for variables to display specific regions of interest. For all four types of graphs, it is possible to set a window on the $x$-variable, the $y$-variable, and the density. For isotherms and isochores, it is also possible to set a window on the temperature.

In plotting the curves, you may use the table values of density and temperature or specify other values so that interpolations are done to obtain the curve points. For all four types of graphs, the default is to use consecutive table values for both density and temperature. For isotherms, you may specify the number of density points to be plotted. Here interpolations are done and the density points are equally spaced between the minimum and maximum values. If the $x$-axis is $\log$, the density poirts are equally spaced after the $\log$ is taken. Also, for isotherms, a step size may be specified for the temperature table enabling the use of nonconsecutive table values. In addition, you may list individual temperature points to be plotted so that interpolations are done. For isochores, the temperature-density options are just the reverse of isotherms. That is, for temperature, you may specify the number of points to be used and, for density, you can use nonconsecutive table points or list specific density points to be used. For Hugoniots and adiabats, you may specify the number of density points to be plotted. Here only the density variable is relevant because you do not have an incipendent temperature variable.

\section{PROMPIS AND COMMANDS}

This section lists and describes in detail the prompts and commands used by SES2D.

A. Prompts

INPUT DATA?

MORE?

CHANGES?
This is the initial prompt and the prompt given after a response of YES to the prompt NEW GRAPH?. The code expects a list of commands specifying a graph. Any daia for previous graphs that may be in internal tables are wiped out. The code starts from scratch in constructing the internal iables for the new graph.

This prompt wili be given after an INPUT DATA? or CHANGES? prompt but oniy if the DN= command has been absent frori the command string for that prompt. Because. $D N=$ serves as a command to piot the data strings in the internal tables, the code is assuming from the absence of $D N=$ that yoi wish to add more to the internal tables before plotting. Therefore, you may give some more plot commands or merely give $\mathrm{DN}=$.

This prompt is given after a graph has been plotted. You may at this time delete curves from the existing data strings (using DC commands) or add to the existins data strings of the internal tables by typing in plot conmands. The relevant parts of the internal tables are retained for the next plot. If the sanie graph is desired on another output device, just type $D N=$ and hit carriage return. If you do wish to wipe clean the internal tables and go on to the next graph, you have two options. You hit return and wail for the NEW GRAPH? prompt. With a YES 
NEW GRAPH? response to the NEW GRAPH? prompt, you cycle back to INPUT DATA?. Alternatively, after CHANGES?, you cain type in your command string for the new graph with the command NEXT also in the command string. This will avoid having both the NEW GRAPH? and INPUT DATA? prompts being given.

The code is asking if you want another graph or if you wish to terminate the session. YES will lead to the next graph. Ainy other response will terminate the session.

The remaining prompts are different in that the code gives them only when a particular piece of data is needed.

MN?

LB?

SES?

DN?
The code needs a material number. Type the number immediately after the ?.

This prompt gives you the opportunity to change from one library to another. After the ?, type the new library name. If you switch libraries and attempt to get the same material, the code will appear to do it but in reality will not. Once a given material is obtained from tables and is in the internal tables of the code, it is not read in again.

This prompt givez jou the opportunity to change from one SESAME format to anoticer (such as from SESAME1 to SESAME2). Just after the ?, type i for SESAMEl or 2 for SESAME2. If you switch formats and attempi to get the same material, the code will appear to do it but in reality sill not. Once a given material is obtained from tables and is in the internal tables of the code, it is not read in again.

The code is in a situation where it needs the output device number. Give it. This only arises if you respond to a MORE? prompt with the command NEXT. This implies that you have data in the internal tables that have never been plotted. You are about to wipe out this data because of the command NEXT. Before destroying the present internal tables, the code plots them on the output device given after the DN? prompt.

The code has the capability to request that you re-enter a command if the code does not recognize something you have entered. In this circumstance, prompts will be written that are self-explanatory.

\section{B. Commands}

Commands are entered with blank delimiters after the first four prompts. The order of the commands is completely arbitrary. Most commands have defaults. Exceptions are $D N=$ and, in some circumstances, the commands specifiying the type of graph; for example, $\mathrm{p}(\mathrm{rho}) \mathrm{t}, \mathrm{E}(\mathrm{t}) \mathrm{h}$, eic. $\mathrm{DN}=$ serves to tell the code the output device you want, and, also, by its presence in a command string, it indicates that you want the 
internal tables plotted. That is, its absence or presence instructs the code to not plot or to plot. The graph specification has a default except when plot commands are given for the CHANGES? and MORE? prompts. In those two cases, if no graph specification is given, all plot commands are ignored. The logic is that the specification [for example, $p(r h o) t$ ] serves both as an add curve command and as an indicator of the type of graph. The first group of commands listed below are valid for all types of graphs. The four groups of commands that follow are specific to isotherms, isochores, Hugoniots, and adiabats.

\section{General Commands}

$\mathrm{LB}=\mathrm{NAME}$

$\mathrm{MN}=$ INTEGER

SES*

NT =INTEGER

$\mathrm{NR}=$ INTEGER

DN=INTEGER

LINX LINY

LOGX LOGY

YGRD NGRD

YDLUT NDULT
Specifies a library name. The default is SESAME for the open partition and SESAMEA for the closed partition.

Specifies a material number. There is no default upon the first attempted use of a material number. A MN? prompt will be given if $\mathrm{MN}=$ has not been previously entered. After a material number has been entered, the default is to the last used value.

Specifies the format of the tables to be plotted. If * is 1 , SESAME1 is used. If * is 2 , SESAME2 is used. Initial default is SESAME2. Subsequent defaults are to the last used format.

These commands specify the maximum sizes of the SESAMEl tables. NT= gives the maximum number of temperatures and $\mathbf{N R}=$ gives the maximum number of etas. Initial defaults are $\mathrm{NT}=25$ and $\mathrm{NR}=100$. Susequent defauits are to the last used values.

Specifies the output device: 1 for microfilm, 2 for microfiche, and 3 for Tektronix. No default ever.

Specifies type of axis: linear $x$-axis, linear $y$-axis, $\log x$-axis, $\log$ $y$-axis. Initial default is $\log -\log$. Susequent defaults are to the last used axes.

Specifies if there is to be a grid. YGRD is yes, NGRD is no. Initial default is no. Subsequent defaults are to the last used specification.

There is a routine in the program that thins the points to be plotted. That is, any points that are superfluous with respect to the graph resolution are eliminated. YDLUT commands that the thinning be done. NDLUT commands that it not be done. Initial default is YDLUT. Subsequent deíaults are to the last used procedure.

If this response is given to the NEW GRAPH? prompt, the code cycles back to the INPUT DATA? prompt. Any other response to NEW GRAPH? terminates the session. 
NEXT

QUIT or END

DC'N

RESET

2. Isotherm Commands

NPS $=$ INTEGER

TABPT

T=REAL NUMBER

NSTEP $=$ INTEGER
When included in the command string for either the MORE? or CHANGES? prompt, this command effectively converts these prompts into the INPUT DATA? prompt.

This command typed after any of the above prompts causes the program to terminate.

- is any letter, a through $\mathbf{z} . \mathbf{N}$ is an integer, 1 through 26 . This command deletes curve * and the succeeding $\mathrm{N}-1$ curves from the graph. It is to be used only with the CHANGES? prompt. $\mathbf{N}$ can be left off the command; then $N=1$. To command that the new graph be produced, you must give a $\mathrm{DN}=$ command.

This command can only be given in the command string of the INPUT DATA? prompt or the CHANGES? and MORE? prompts when a NEXT command is also present. The command causes all defaults to be reset to their initial values; that is, the status of the program is as if you had just started the program running. (The exceptions are $\mathrm{LB}=$ and $\mathrm{MN}=$; they are not reset but retain their last used values.) Unless you have something specific in mind, it is probably good to give the RESET command whenever the material number is changed.

The integer gives the number of density points to be used for a curve. When the NPS= command is given, interpolations are done to obtain the curve points. The density points are equally spaced between RL and RU. If the $x$-axis is log, the density points are equally spaced after the $\log _{\mathrm{g}}$ is taken.

To go from the NPS= mode back to using only the density points in the table, give a command TABPT. The initial default for NPS = and TABPT is TABPT. Susequent déaults are to the last used procedure. If no $T=$ command is given and one is in the TABPT mode, only table temperatures and densities are used, and no interpolations are done for $\mathrm{p}$ and $\mathrm{e}$. Interpolations must always be done to obtain pdt. pdr, edt, and edr.

If you want other than a table temperature, type in the temperature in degrees kelvin. More than one $T=c a n$ be given. If $T=$ is given, no table temperatures are used. If no $T=$ 's are given, the temperatures are taken from the table. If $T=$ 's are given, interpolations are done.

If table temperatures are being used, NiTEP enables you to pick the interval used to space through ine table. NSTEP $=1$ takes all table values, NSTEP $=2$ takes every second value, etc. One is the initial default. Subsequent defaults are to the last used value. 
RL=REAL NUMBER

RU $=$ REAL NUMBER

$T L=$ REAL NUMBER

TU= REAL NUMBER

YL=REAL NUMBER

$Y U=R E A L ~ N U M B E R$

$X L=R E A L ~ N U M B E R$

$\mathrm{XU}=\mathrm{REAL}$ NUMBER

3. Isochore Commands

RNPS $=$ INTEGER

RTABPT
It is possible to set a window on both density (lower density, RL, and upper density, RU) and temperature (lower tenperature, $T L$, and upper temperature, TU). The initial defaults are to the table maxima and minima. Subsequent defaults are to the last used values. An exception to this arises if any last used values fall outside the table ranges. Then those window limits are set to their respective table limits. If you attemot to set a limit outside of a table's range, the code resets the limit to the table limit. Units are megagrams per meter cubed for densities and degrees kelvin for temperatures. If you are doing a graph with a log $\mathrm{x}$-axis and $\mathrm{RL}$ is less than or equal to $0.0, \mathrm{RL}$ is reset to $1.0 \mathrm{e}-10$.

It is possible to set a window on the y-variable (lower limit, YL, and upper limit, YU). The same bounds apply to all $y$-variabses even though the units are not the same. The initial defaults are to $Y L=1 . e-3$ and $Y U=1 . e 19$. Subsequent defaults are to the last used values. If the $y$-axis is $\log , Y L$ is set to 1.e- 3 if $Y L$ is less than or equal to 0 . If the $y$-axis is log, a check is made to see if $\mathrm{YU}$ is greater than 1.e22* $\mathrm{YL}$ or less than $\mathrm{YL}$. If it is greater than 1.e22*YL or less than YL, YU is set to $1 . e 22^{*} \mathrm{YL}$. These checks are done whether the routine is defaulting to previous values or the user is typing in new values. The units are those appropriate for the $y$-axis variable.

These limits specify a window on the variable being plotted on the $\mathrm{x}$-axis. The discussion is the same as just given for the $y$-axis window.

The integer gives the number of temperature points to be used for a curve. When the RNPS= command is given, interpolations are done to obtain the curve points. The temperature points are equally spaced between RTL and RTU. If the x-axis is log, the temperature points are equally spaced after the $\log$ is taken.

To go from the RNPS= mode back to using only the temperature points in the table, give a command R TABPT. The initial default for RNPS= and RTABPT is RTABPT. Subsequent defaults are to the last used proceciur. \&. ... $R=$ command is given and you are in the RTABPT mode, only table densities and temperatures are used and no interpolations are done for $p$ and $e$. Interpolations must always be done to obtain pdt, pdr, edt, and edr. 
R=REAL NUMBER

RNSTEP $=$ INTEGER

RRL=REAL NUMBER

RRU=REAL NUMBER

RTL=REAL NUMBER

RTU=REAL NUMBER

RYL=REAL NUMBER

RYU=REAL NUMBER

RXL $=$ REAL NUMBER RXU $=$ REAL NUMBER
If you want other than a table density, type in the density in megagrams per meter cubed. More than one $R=$ can be given. If $R=$ is given, no table densities are used. If no $R==$ 's are given, the densities are taken from the table. If $\mathbf{R}=$ 's are given, interpolations are done.

If table temperatures are being used, RNSTEP enables you to pick the interval used to space through the tabie. $R$ NSTEP $=1$ takes all table values, $R$ NSTEP $=2$ takes every second, etc. One is the initial default. Subsequent defaults are to the last used value.

It is possible to set a window on both the density (lower density, RRL, and upper density, RRU) and temperature (lower temperature, RTL, and upper temperature, RTU). The initial defaults are to the tatle maxima and minima. Subsequent defaults are to the last used values. An exception to this arises if any last used values fall outside the table ranges. Then those window limits are set to their respective table limits. If you attempt to set a limit outside of a table's range, the code resets the limit to the table limit. Units are megagrams per meter cubed for densities and degrees kelvin for temperatures. If you are doing a graph with a $\log x$-axis and RTL is less than or equal '? 0.0, RTL is reset to $1.0 \mathrm{e}-10$.

It is possible to set a window on the $y$-variable (lower limit, RYL, and upper limit, RYU). The same bounds apply to all $y$-variables even though the units are not the same. The initial defaults are to $R Y L=1 . e-3$ and $R Y U=1 . e 19$. Subsequent defaults are to the last used values. If the $y$-axis is $\log , R Y L$ is set to $1 . e-3$ if RYL is less than or equal to 0 . If the $y$-axis is $\log$, a check is made to see if RYU is greater than 1.e22*RYL or less than RYL. If it is greater than 1.e22*RYL or less than RYL, RYU is set to I.e22* $R Y L$. These checks are done whether the routine is defaulting to previous values or the user is typing in new values. The units are those appropriate for the $y$-axis variable.

These limits specify a window on the variable being plotted on the $x$-axis. The discussion is the same as just given for the $y$-axis window, 


\section{Hugoniot Commands}

HNPS $=$ INTEGER

HTABPT

YCONS

NCONS

HRHO0 $=$ =REAL NUMBER

HE0 $=$ REAL NUMBER

HPO $=$ REAL NUMBER

HRL $=$ REAL NUMBER

HRU $=$ REAL NUMBER
The integer gives the number of density points to be used for a curve. The density points are equally spaced between HRL anc. HRU. If the $x$-axis is log, the density points are equally spaced after the log of rho is taken.

To go from the HNPS= mode back to using only the density points in the table, give a command HTABPT. The initial default for HNPS= and HTABPT is HTABPT. Subsequent defaults are to the last used procedure.

To determine a Hugoniot, you must specify initial shock conditions denoted by HRHOO, HEO, and HPO for initial density, initial internal energy, and initial pressure, respectively. If you impose thermodynamic consistency among these variables, you need only input two variables, in particular, HRHOO and HEO for this code. However, for some uses (foams), all three variables, HRHOO, HEO, and HPO, are needed without thermodynamic consistency. Use YCONS and NCONS to switch between the two modes. YCONS is the initial default and means that $\mathrm{HPO}$ is to be determined from $\mathrm{HRHOO}$ and $\mathrm{HEO}$ in a thermodynamically consistent way. NCONS means that HRHOO, HEO, and HPO are independent of each other. Defaults other than the initial are to whatever was last used.

These commands give the initial parameters for the Hugoniot. HRHOO is the initial density in megagrams per meter -ubed, HEO is the initial internal energy in megajoules per kilogram, and HPO is the initial pressure in gigapascals. More than one group of such commands can be given. The code takes the commands in order. The initial defaults are HRHOO=rho? (from the SESAME table), $\mathrm{HEO}=0.0$, and $\mathrm{HPO}=0.0$. Subsequent defaults are to last used values. HRHOO cannot be set outside the bounds of the table and will be reset to the lower table bound if it is outside. If YCONS is on, HPO is always calculated from HRHOO and HEO, and any HPO= command is ignored.

It is possible to set a window on the density (lower density, HRL, and upper density, HRU). The initial defaults are HRL= rho0 and HRU $=\min \left(2.5^{*} \mathrm{RHOO}\right.$, upper table limit). (rho0 is from the SESAME table.) Subsequent defaults are to the last used values. Note that HRL is set to HRHOO if HRL is less than HRHOO, and HRU is set to the table maximum if HRU is greater than the table maximum. Units are megagrams per meter cubed. 
HYL=REAL NUMBER

HYU $=$ REAL NUMBER

HXL=REAL NUMBER

HXU $=$ REAL NUMBER

HERR

HNERR

5. Adiabat Commands

SNPS $=$ INTEGER

STABPT

SRHOO=REAL NUMBER SEO $=$ REAL NUMBER
It is possible to set a window on the y-variable (lower limit, HYL, and upper limit, HYU). The same bounds apply to all $y$-variables even though the units are not the same. The initial defaults are to $H Y L=1 . e-3$ and $H Y U=1 . e 19$. Subsequent defaults are to the last used values. If the y-axis is log, HYL is set to $1 . e-3$ if HYL is less than or equal to 0 . If the $y$-axis is $\log$, a check is made to see if HYU is greater than 1.e22*HYL or less than HYL. If it is greater than I.e22*HYL or less than HYL, HYU is set to $1 . e 22^{*} \mathrm{HYL}$. These checks are done whether the routine is defaulting to previous values or the user is typing in ner, salues. The units are those appropriate for the $y$-axis variable.

These limits specify a window' on the variable being plotted on the $\mathrm{x}$-axis. The discussion is the same as just given for the $y$-axis window.

The routine $\mathbf{t}^{\prime}$ at calculates Hugoniot parameters can have difficulties, in particular, at rho=HRHOO and for large rho. A message is printed if the routine is having difficulty. If you want to know the problem densities, give the command HERR. The problem densities will be printed if you repeat the calculation. HNERR turns off the printing. The initial default is HNERR. Subsequent defaults are to what was last done.

The integer gives the number of density points to be used for a curve. The density points are equally spaced between SRL and SRU. If the $x$-axis is $\log$, the density points are equally spaced after the $\log$ of rho is taken.

To go from the SNPS= mode back to using only the density points in the table, give a command STABPT. The initial default for SNPS= and STABPT is STABPT. Subsequent defaults are to the last used procedure.

These commands select the desired adiabat. The adiabat drawn will go through SRHOO and SEO, where SRHOO is a density in megagrams per meter cubed and SEO is an energy in megajoules per kilogram. More than one group of such commands can be given. The code takes the commands in order. The initial defaults are SHROO= rhoO (from the SESAME table) and $\mathrm{SEO}=0.0$. Subsequent defaults are to last used values. SRHO0 cannot be set outside the bounds of the table and will be reset to 
SRL=REAL NUMBER

SKU $=$ REAL NUMBER

SYL-REAL NUMBER

SYU=REAL NUMBER

SXL $=$ REAL NUMBER

SXU $=$ REAL NUMBER

SERR

SNERR the lower table bound if it is outside. There is no freedom to select SPO as in the Hugoniot mode.

It is possible to set a window on the density (lower density, SRL, and upper density, SRU). The initial defaults are $S R L=r h o 0$ and $S R U=\min \left(2.5^{*}\right.$ rho0, upper table limit). (rho0 is from the SESAME table.) Subsequent defaults are to the last used values. Note that SRL is set to the lower table linit if SRL is less than the lower table limit, and SRU is set to the table maximum if SRU is greater than the table maximum. Units are megagrams per meter cubed.

It is possible to set a window on the y-variable (lower limit, SYL, and upper limit, SYU). The same bounds apply to all y-variables even though the units are not the same. The initial defaults are to $S Y L=1 . e-3$ and $S Y U=1 . e 19$. Subsequent defaults are to the last used values. If the $y$-axis is $\log$, SYL is set to $1 . e-3$ if SYL is less than or equal to 0 . If the $y$-axis is $\log$, a check is made to see if SYU is greater than $1 . e 22^{*} \mathrm{SYL}$ or less than SYL. If it is greater than 1.e22*SYL or less than SYL, SYU is set to $1 . e 22 * \mathrm{SYL}$. These cherks are done whether the routine is defaulting to previous values or the user is typing in new values. The units are those afpropriate for the $y$-axis variable.

These limits specify a window on the variable being plotted on the $\mathbf{x}$-axis. The discussion is the same as just given for the $\mathbf{y}$-axis window.

The routine that calculates adiabat parameters can have difficulties. A message is printed if there is a problem. If you want to know the problem densities, give the command SERR. The problem densities will be printed if you repeat the calculation. SNERR turns off the printing. The initial default is SNERR. Subsequent defaults are to what was last done.

\section{EXAMPLES}

Figures 1 and 2 show a sample run with a number of graphs. The first page has the initial input for the run. This input (material number and device number) is the minimum amount of information needed to construct a graph. All other parameters are defaulted. In the following pages, the commands given after CHANGES? on a graph are the input that produces the next graph. For this run, we are working with a public file SESAME and a local file SES for the equation of state libraries.

A few words describing the graphs are in order. All is obvious except the table on the left side of the graphs. The table headings contain the material numbers of materials being presented; contractions of the words isotherm, isochore, Hugoniot, or adiabat describe the types of curves shown. The 1 or 2 following 
the contracted descriptor denotes SESAMEI or SESAME2 format. Under the headings are labels for the individual curves being plotted. For isotherms these are temperatures; isochores will have densitites; Hugoniots will list HEO; and adiabats will list SEO. The user has to retain HRHOO, SRHO0, and HPO; they are not listed on the graphs. 
sedud 18.8

tof toso soald ar

eurront llbrory to soseme

ingut dete dn-3

Fig. I. Initial irput to construct a graph. 


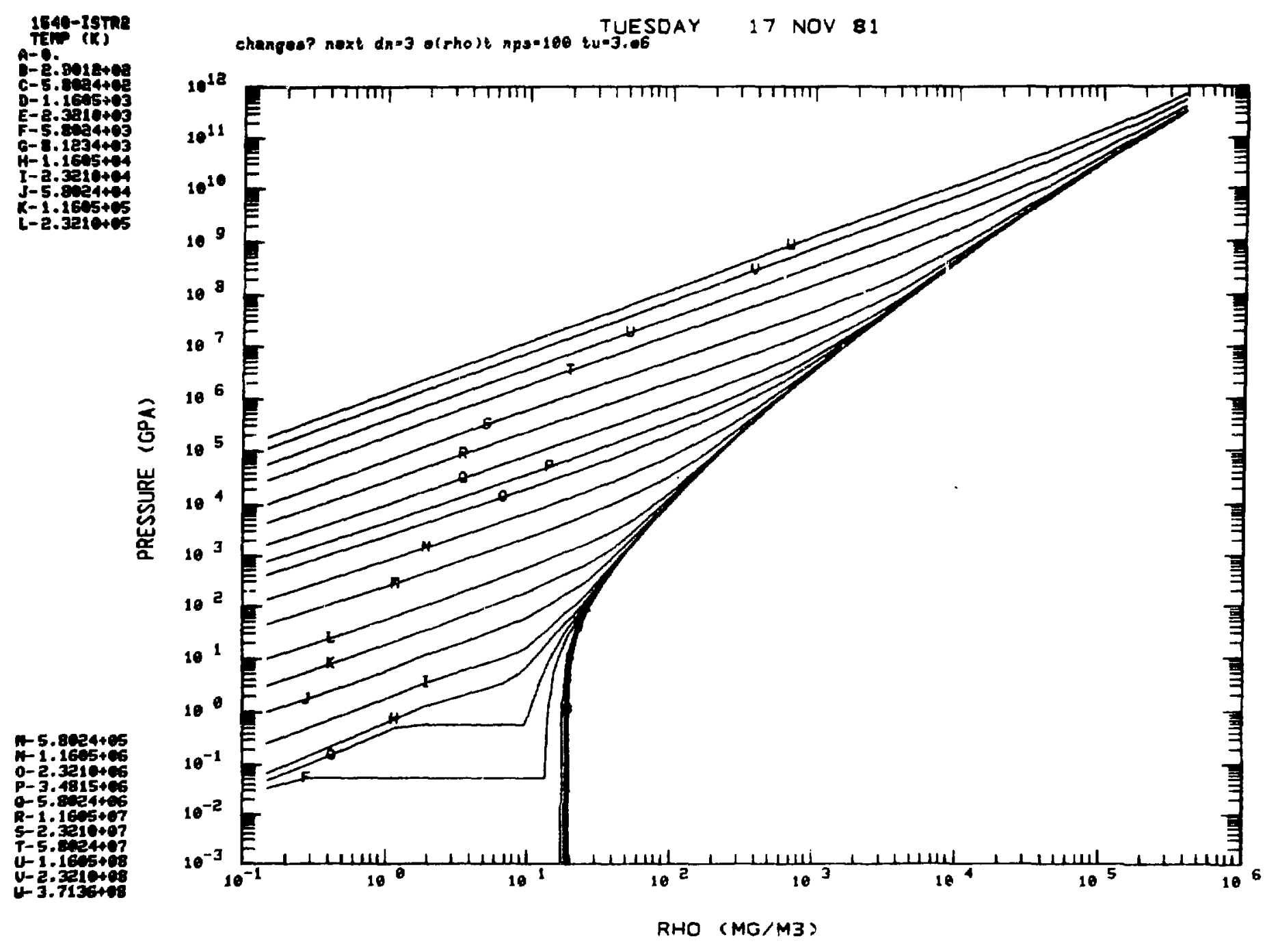

T-4 LTSS SESED 49 


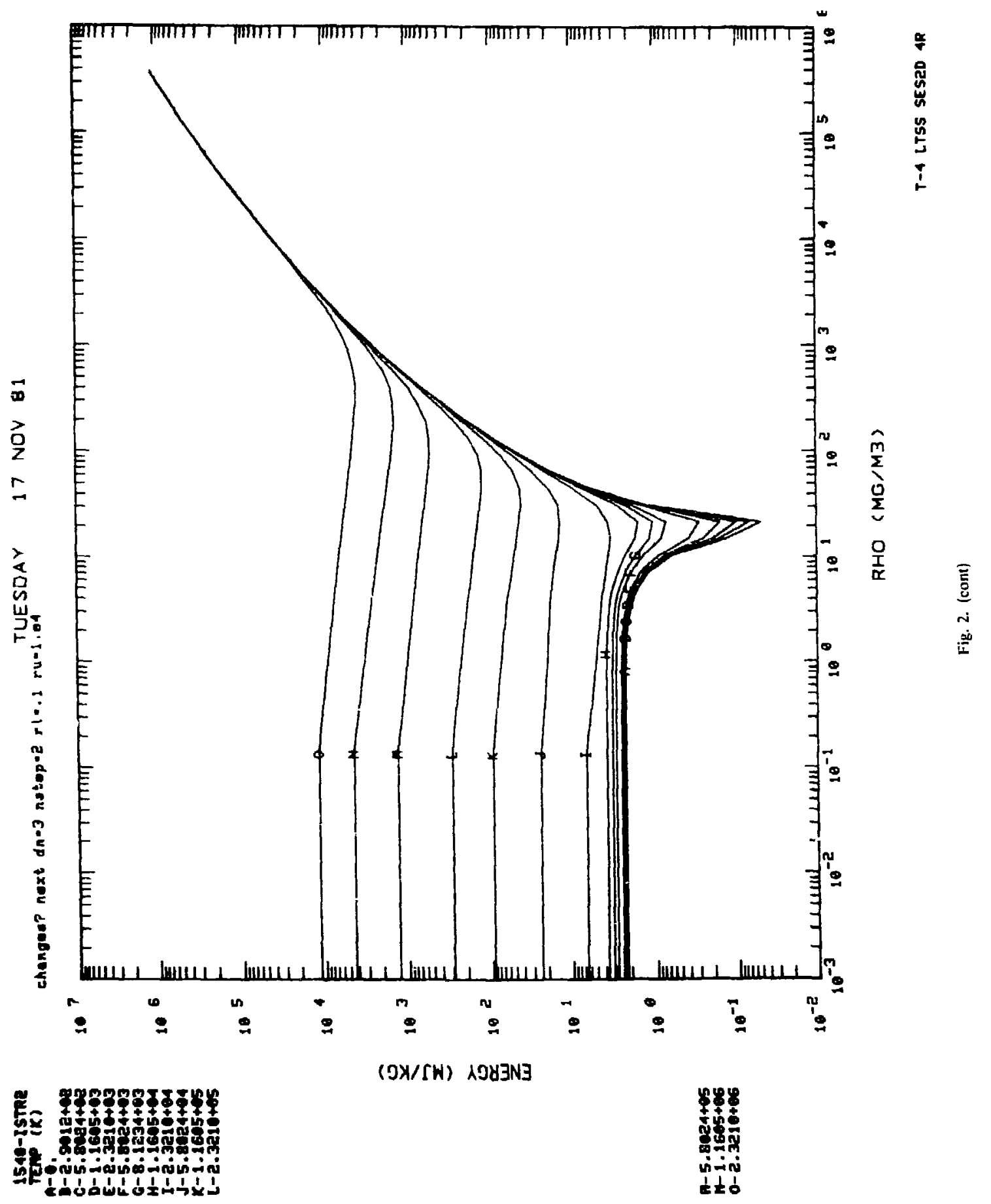



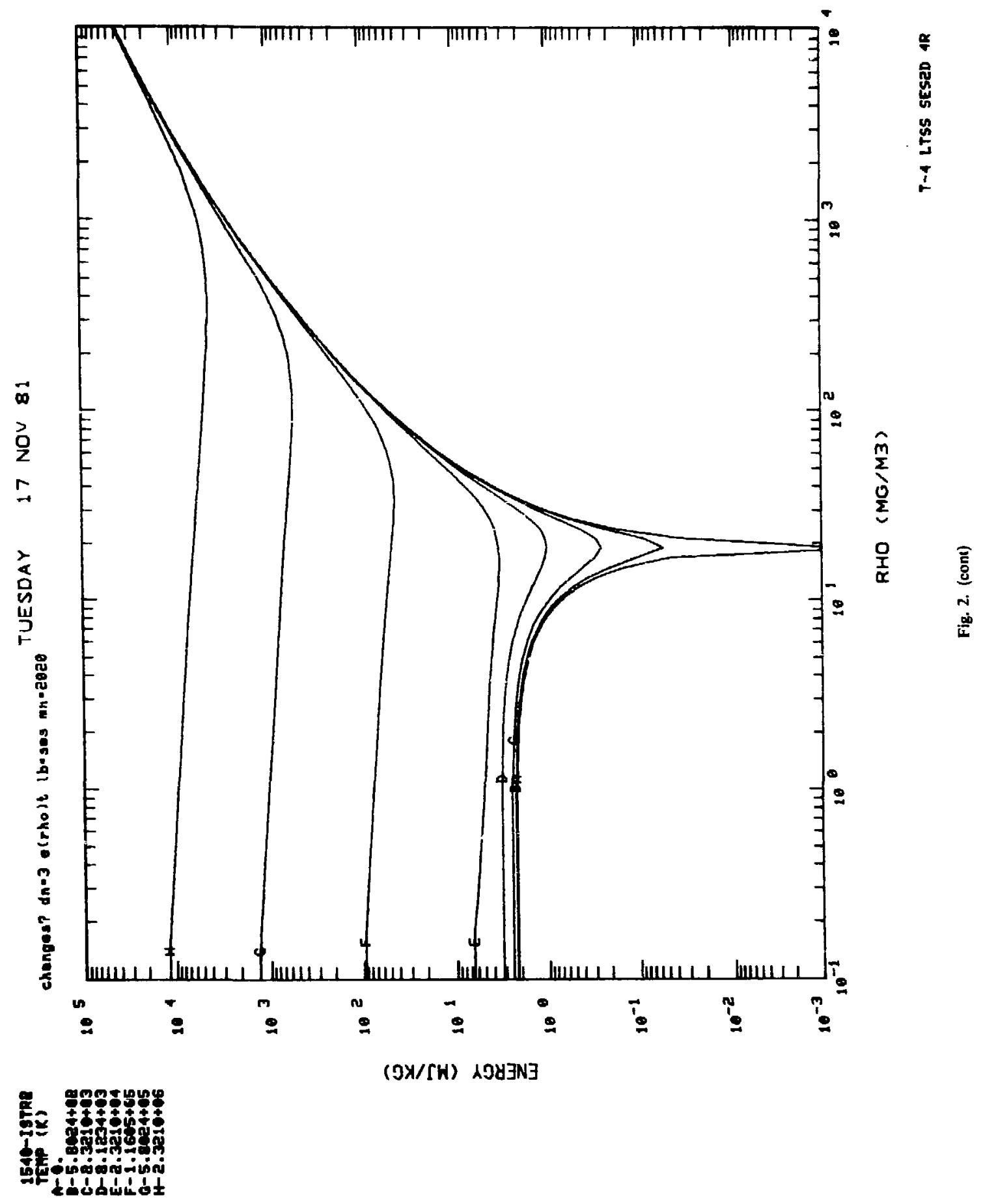


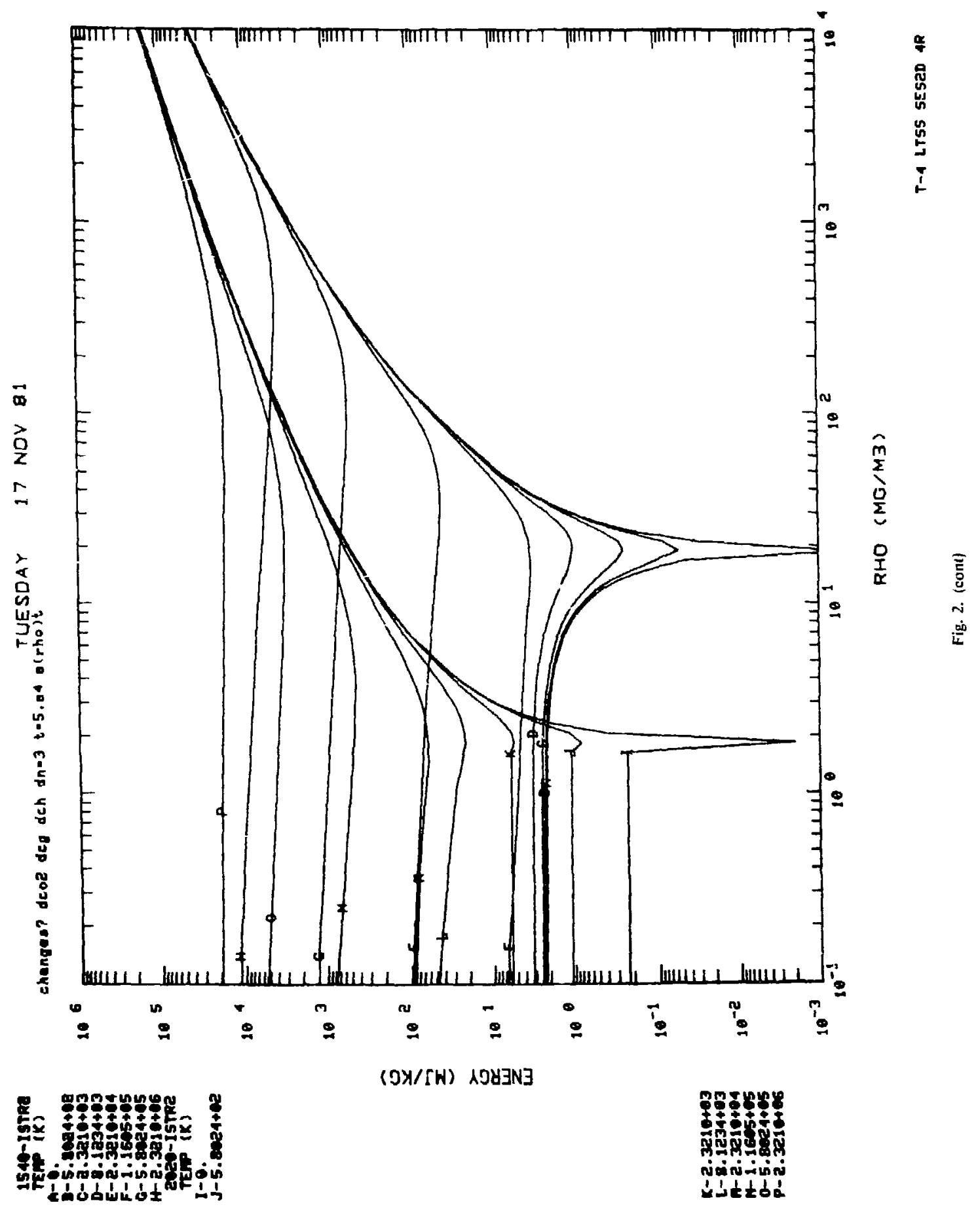



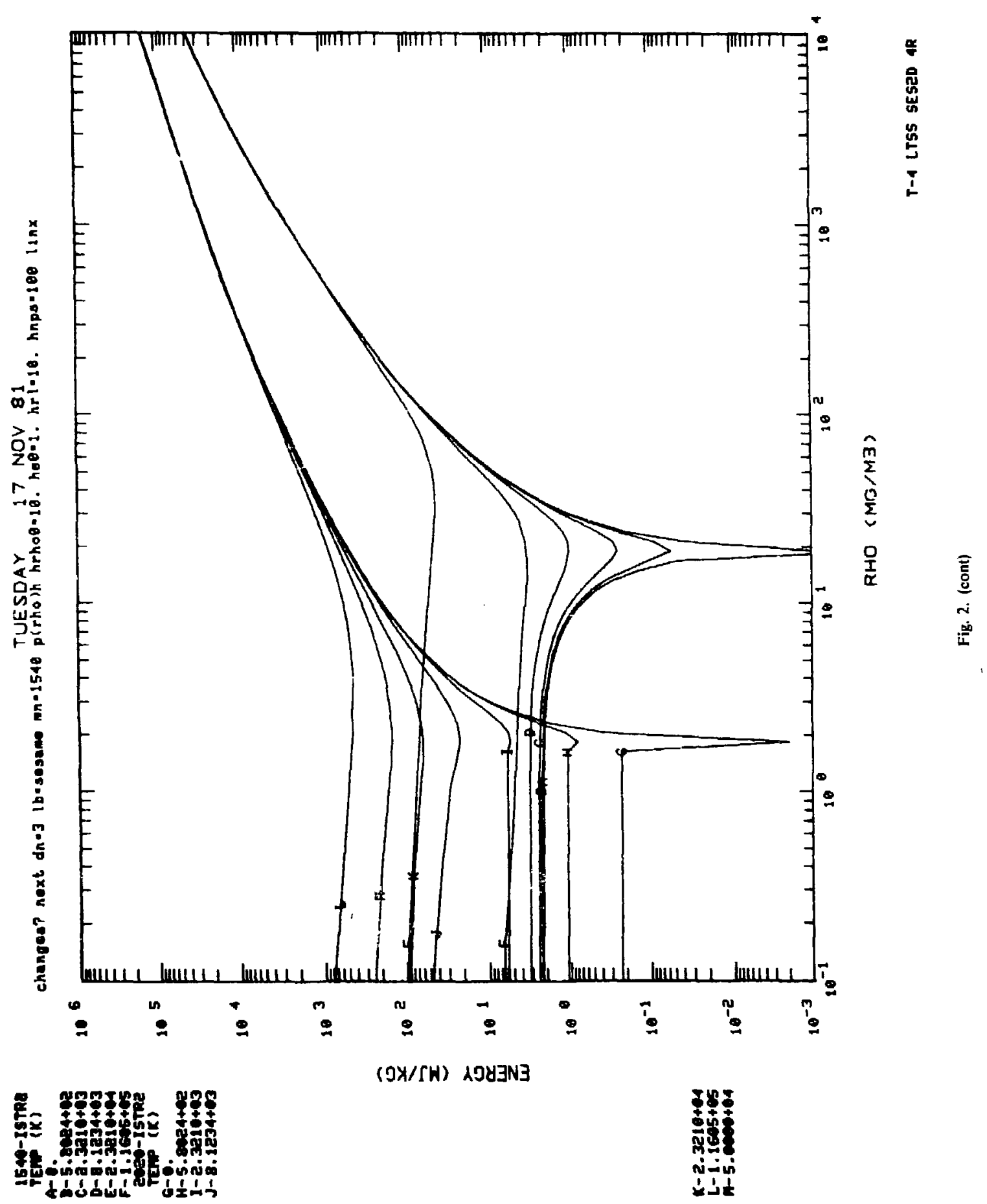


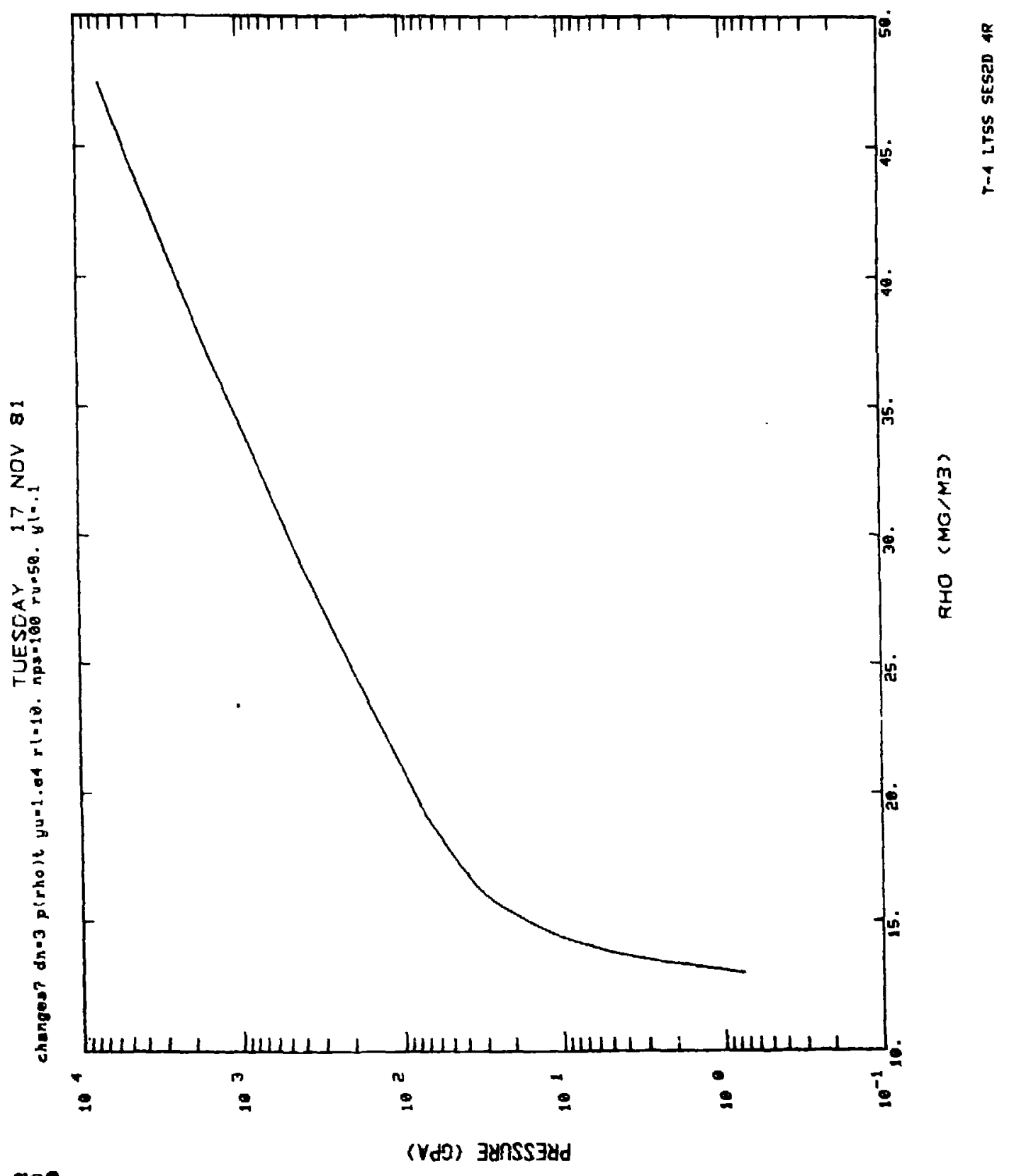



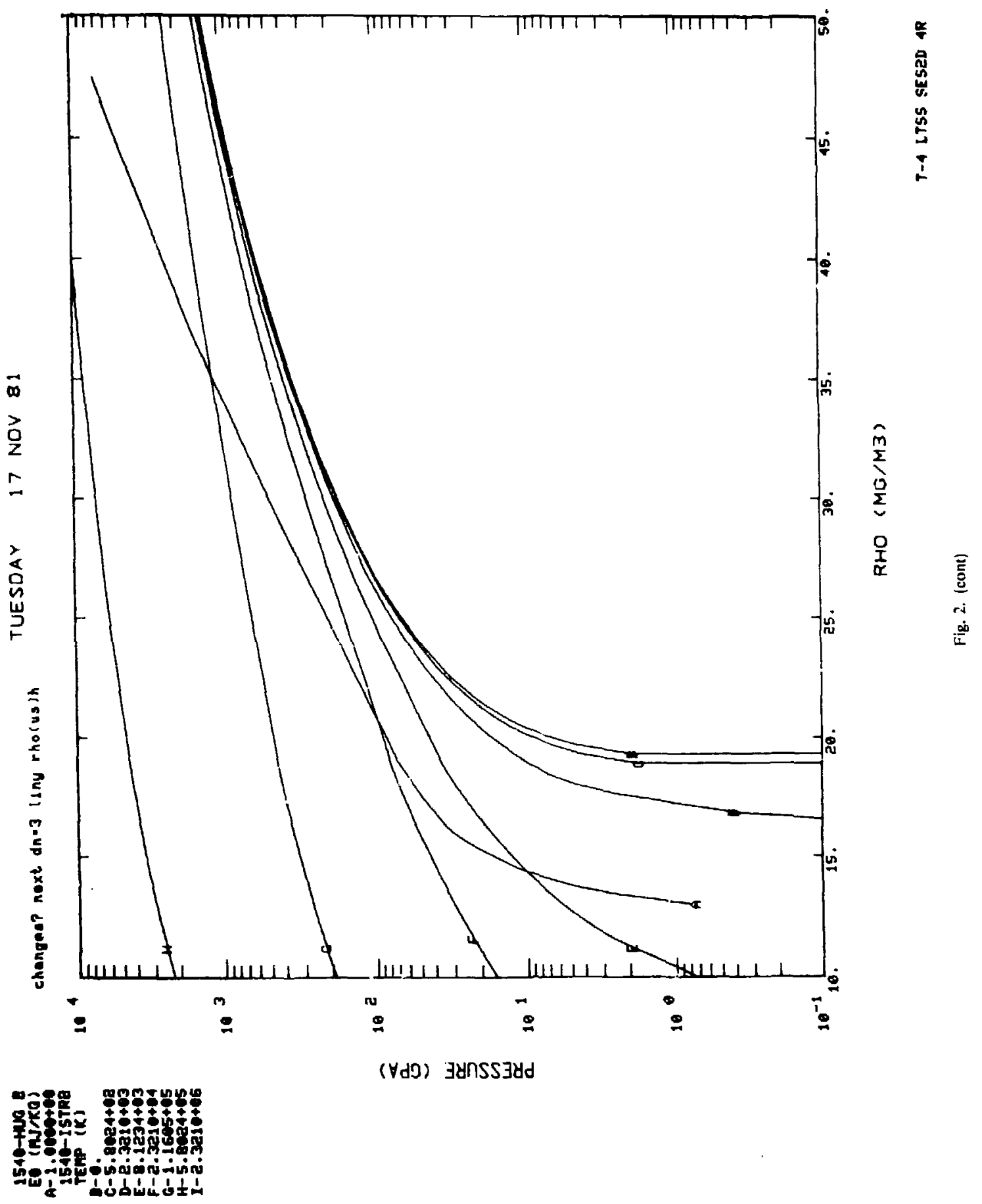
$\tilde{\sigma}$

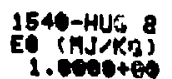

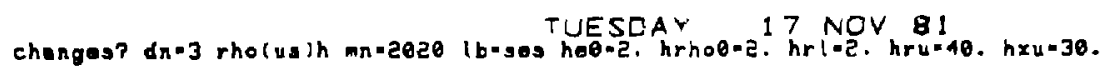

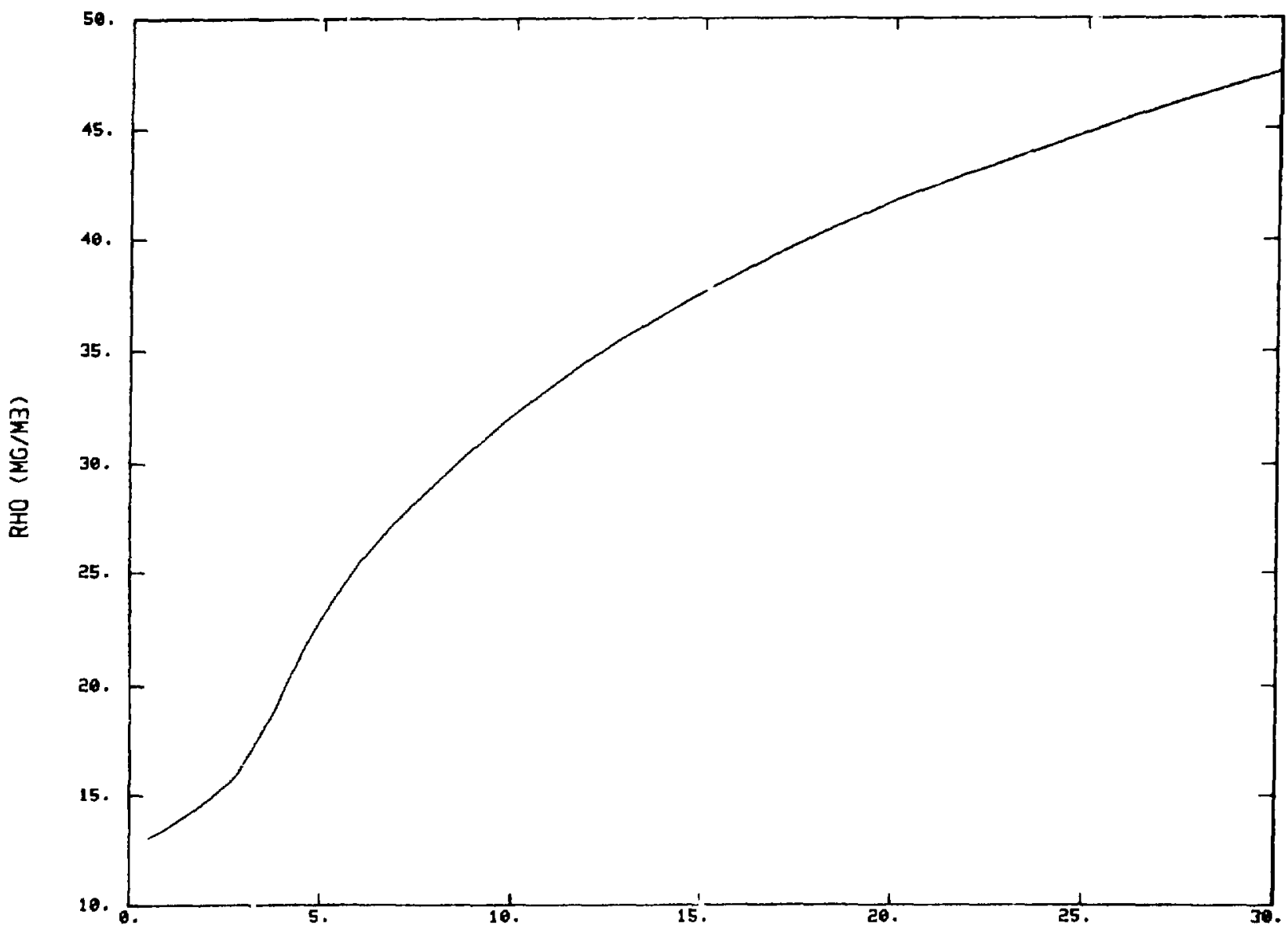

US (KM/SEC)

T-4 LTSS SESED AR

Fig. 2. (cont) 


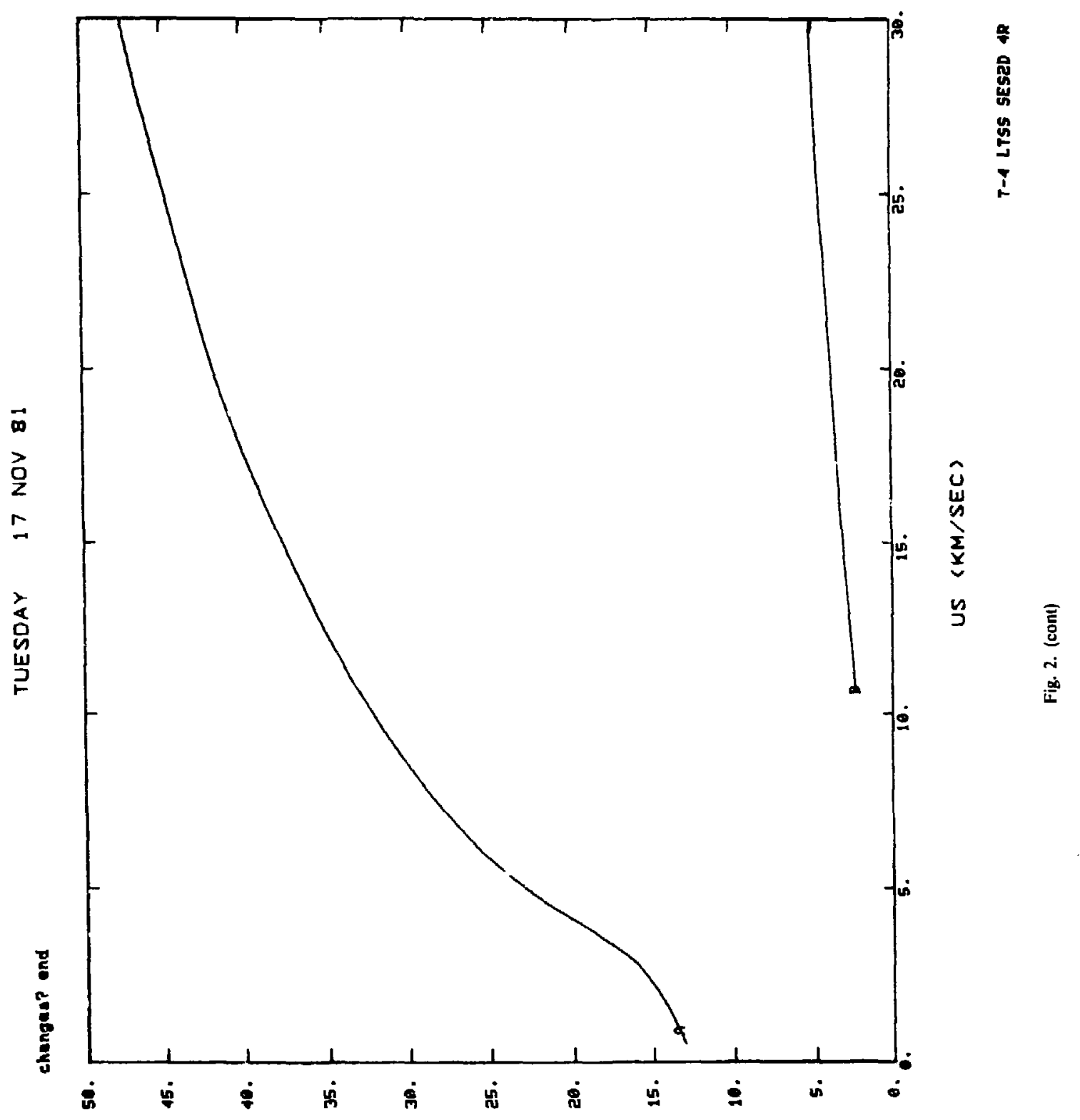

(EW/OW) OHY

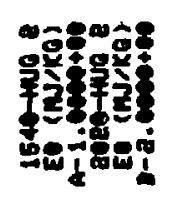


LA- $-9164-M$

DE82 014120

\section{SES2D User's Manua!}

James D. Johnson

Stanford P. Lyon

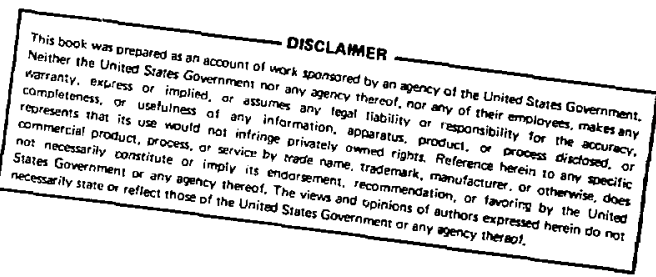


This work was supported by the US Department of Energy, Division of Materials Sciences, Office of Energy Research.

Edited by Kyle Thom

Photocomposition by Pandin H. Mayne

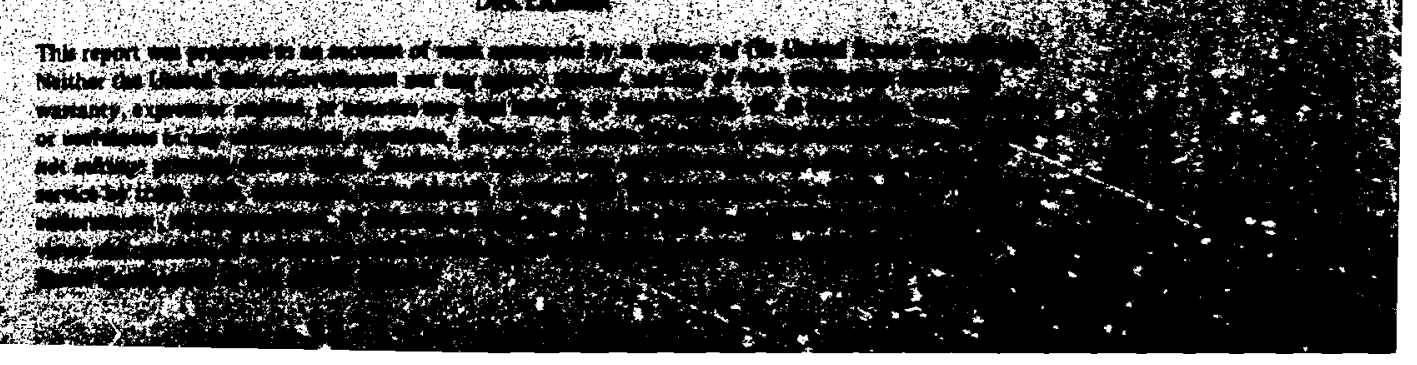


SES2D User's Manual

by

James D. Johnson and Stanford P. Lyon

\begin{abstract}
SES2D is an interactive graphics code designed to generate plots of equation of state data from the Los Alamos National Laboratory Group T-4 computer libraries. This manual discusses the capabilities of the code. It describes the prompts and commands and illustrates their use with a sample run.
\end{abstract}

\title{
1. GENERAL OVERVIEW
}

SES2D is an interactive graphics code designed to generate plots of equation of state data. Versions of SES2D for use with sequential and random libraries exist on both the 7600 and Cray computers. In response to code prompts, you may specify a library name, material number, output device, and various parameters associated witi the format and type of graph. The default library is SESAME for the open partition and SESAMEA for the closed partition. The output devices are microfilm, microfiche, and the Tektronix terminal. The code will continue to prompt you for more input until the output device parameter is entered. In addition to the type of curve, you may specify linear or logarithmic axes either with or without grid lines. Here the initial default is to $\log -\log$ axes with no grid lines. Once a curve or family of curves has been plotted, you may then modify the existing plot (for example, by deleting a specific curve or curves from a family of curves). Furthermore, curves may be added to the same grid to compare two or more materials.

Currently, there aie four classes of graphs: isotherms, isochores, Hugoniots, and adiabats. To select a particular graph, type a command of the form $y(x) z$, where $z=t$ for isotherms, $z=r$ for isochores, $z=h$ for Hugoniots, and $z=s$ for adiabats. The variable $x$ is plotted on the abscissa and the variable $y$ is plotted on the ordinate. The initial default is to plot pressure isotherms $p(r h o) t$; subsequent defaults are to the last type of graph plotted. What is allowed for $x$ and $y$ depends on which class of graph is being plotted. The variables allowed for each class of graph are listed below. Any pair of values may be used for $x$ and $y$.

ISOTHERMS $\quad \mathrm{p}$ e rho pdt pdr edt edr eta $v$

ISOCHORES $\quad p \quad e \quad t$ pdt $p d r$ edt edr eta $v$

HUGONIOTS $p$ e rho $t$ eta $v$ us up

ADIABATS $p$ e rho $t$ eta $v$ up $c$

Here $p$ is pressure; $e$ is energy; rho is density; $t$ is temperature; $p d t$ is the temperature derivative of $p$; pdr is the density derivative of $p$; edt is the temperature derivative of $e$; edr is the density derivative of $e$; eta is rho/rho0, where rho0 is the reference density given in the SESAME table; $v$ is $1 /$ rho; us is the shock 
velocity; up is the particle velocity; and $\mathrm{c}$ is the sound velocity. In plotting a curve, $v$ is set equal to $1.0 \mathrm{e} 100$ if rho is less than or equal to zero.

Specific upper and lower limits can be set for variables to display specific regions of interest. For all four types of graphs, it is possible to set a window on the $x$-variable, the $y$-variable, and the density. For isotherms and isochores, it is also possible to set a window on the temperature.

In plotting the curves, you may use the table values of density and temperature or specify other values so that interpolations are done to obtain the curve points. For all four types of graphs, the default is to use consecutive table values for both density and temperature. For isotherms, you may specify the number of density points to be plotted. Here interpolations are done and the density points are equally spaced between the minimum and maximum values. If the $x$-axis is $l o g$, the density points are equally spaced after th $\log$ is taken. Also, for isotherms, a step size may be specified for the temperature table enabling the use of nonconsecutive table values. In addition, you may list individual temperature points to be plotted so that interpolations are clone. For isochores, the temperature-density options are just the reverse of iso'herms. That is, for temperature, you tnay specify the number of points to be used and, for density, yol: can use nonconsecutive table points or list specific density points to be used. For Hugoniots and adiabats, you may specify the number of density points to be plotted. Here only the density variable is relevant because you do not have an independent temperature variable.

\section{PROMPTS AND COMMANDS}

This section lists and describes in detail the prompts and commands used by SES2D.

\section{A. Prompts}

INPUT DATA?

MORE?

CHANGES?
This is the initial prompt and the prompt given after a response of YES to the prompt NEW GRAPH?. The code expects a list of commands specifying a graph. Any data for previous graphs that may be in internal tables are wiped out. The code starts from scratch in constructing the internal tables for the new graph.

This prompt will be given after an INPUT DATA? or CHANGES? prompt but only if the $\mathrm{DN}=$ command has been absent from the command string for that prompt. Because. $D N=$ serves as a command to plot the data strings in the intemal tables, the code is assuming from the absence of $\mathrm{DN}=$ that you wish to add more to the internal tables before plotting. Therefore, you may give some more plot commands or merely give $\mathrm{DN}=$.

This prompt is given after a graph has been plotted. You may at this time delete curves from the existing data strings (using DC commands) or add to the existing data strings of the internal tables by typing in plot commands. The relevant parts of the internal tables are retained for the next plot. If the same graph is desired on another output device, just type $\mathrm{DN}=$ and hit carriage return. If you do wish to wipe clean the internal tables and go on to the next graph, you have two options. You hit return and wait for the NEW GRAPH? prompt. With a YES 
NEW GRAPH? response to the NEW GRAPH? prompt, you cycle back to INPUT DATA?. Alternatively, after CHANGES?, you can type in your command string for the new graph with the command NEXT also in the command string. This will avoid having both the NEW GRAPH? and INPUT DATA? prompts being given.

The code is asking if you want another graph or if you wish to terminate the session. YES will lead to the next graph. Any other response will terminate the session.

The remaining prompts are different in that the code gives them only when a particular piece of data is needed.

MN?

The code needs a material number. Type the number immediately after the?.

LB?

This prompt gives you the opportunity to charge from one library to another. After the?, type the new library name. If you switch libraries and attempt to get the same material, the code will appear to do it but in reality will not. Once a given material is obtained from tables and is in the internal tables of the code, it is not read in again.

SES?

This prompt gives you the opportunity to change from one SESAME format to another (such as from SESAME1 to SESAME2). Just after the ?, type 1 for SESAME1 or 2 for SESAME2. If you switch formats and attempt to get the same material, the code will appear to do it but in reality will not. Once a given material is obtained from tables and is in the internal tables of the code, it is not read in again.

DN?

The code is in a situation where it needs the output device number. Give it. This only arises if you respond to a MORE? prompt with the command NEXT. This implies that you have data in the internal tables that have never been plotted. You are about to wipe out this data because of the command NEXT. Before destroying the present internal tables, the code plots them on the output device given after the DN? prompt.

The code has the capability to request that you re-enter a command if the code does not recognize something you have entered. In this circumstance, prompts will be written that are self-explanatory.

\section{B. Commands}

Commands are entered with blank delimiters after the first four prompts. The order of the commands is completely arbitrary. Most commands have defaults. Exceptions are $D N=$ and, in some circumstances, the commands specifiying the type of graph; for example, $\mathrm{p}($ rho $), \mathrm{e}(\mathrm{t}) \mathrm{h}$, etc. $\mathrm{DN}=$ serves to tell the code the output device you want, and, also, by its presence in a command string, it indicates that you want the 
internal tables plotted. That is, its absence or presence instructs the code to not plot or to plot. The graph specification has a default except when plot commands are given for the CHANGES? and MORE? prompts. In those two cases, if no graph specification is given, all plot commands are ignored. The logic is that the specification [for example, $p(r h o) t$ ] serves both as an add curve command and as an indicator of the type of graph. The first group of commands listed below are valid for all types of graphs. The four groups of commands that follow are specific to isotherms, isochores, Hugoniots, and adiabats.

\section{General Commands}

$\mathrm{LB}=\mathrm{NAME}$

$\mathrm{MN}=\mathrm{INTEGER}$

SES*

NT=INTEGER

NR $=$ INTEGER

$\mathrm{DN}=$ INTEGER

LINX LINY

LOGX LOGY

YGRD NGRD

YDLUT NDULT
Specifies a library name. The default is SESAME for the open partition and SESAMEA for the closed partition.

Specifies a material number. There is no default upon the first attempted use of a material number. A MN? prompt will be given if $\mathrm{MN}=$ has not been previously entered. After a material number has been entered, the default is to the last used value.

Specifies the format of the tables to be plotted. If * is 1 , SESAME1 is used. If * is 2, SESAME2 is used. Initial default is SESAME2. Subsequent defaults are to the last used format.

These commands specify the maximum sizes of the SESAME1 tables. $N T=$ gives the maximum number of temperatures and $\mathrm{NR}=$ gives the maximum number of etas. Initial defaults are $\mathrm{NT}=25$ and $\mathrm{NR}=100$. Susequent defaults are to the last used values.

Specifies the output device: 1 for microfilm, 2 for microfiche, and 3 for Tektronix. No default ever.

Specifies type of axis: linear $x$-axis, linear $y$-axis, $\log x$-axis, $\log$ $y$-axis. Initial default is $\log$-log. Susequent defaults are to the last used axes.

Specifies if there is to be a grid. YGRD is yes, NGRD is no. Initial default is no. Subsequent defaults are to the last used specification.

There is a routine in the program that thins the points to be plotted. That is, any points that are superfluous with respect to the graph resolution are eliminated. YDLUT commands that the thinning be done. NDLUT commands that it not be done. Initial default is YDLUT. Subsequent defaults are to the last used procedure.

If this response is given to the NEW GRAPH? prompt, the code cycles back to the INPUT DATA? prompt. Any other response to NEW GRAPH? terminates the session. 
NEXT

QUIT or END

DC*N

RESET

\section{Isotherm Commands}

NPS=INTEGER

TABPT

T=REAL NUMBER

NSTEP $=$ INTEGER
When included in the command string for either the MORE? or CHANGES? prompt, this command effectively converts these prompts into the INPUT DATA? prompt.

This command typed after any of the above prompts causes the program to terminate.

- is any letter, a through $\mathrm{z} . \mathrm{N}$ is an integer, 1 through 26 . This command deletes curve ${ }^{*}$ and the succeeding $\mathrm{N}-1$ curves from the graph. It is to be used only with the CHANGES? jrompt. $N$ can be left off the command; then $\mathrm{N}=1$. To comnand that the new graph be produced, you must give a $\mathrm{DN}=$ command.

This command can only be given in the command string of the INPUT DATA? prompt or the CHANGES? and MORE? prompts when a NEXT command is also present. The command causes all defaults to be reset to their initial values; that is, the status of the program is as if you had just started the program running. (The exceptions are $\mathrm{LB}=$ and $\mathrm{MN}=$; they are not reset but retain their last used values.) Unless you have something specific in mind, it is probably good to give the RESET command whenever the material number is changed.

The integer gives the number of density points to be used for a curve. When the NPS= command is given, interpolations are done to obtain the curve points. The density points are equally spaced between $R L$ and RU. If the $x$-axis is $\log$, the density points are equally spaced after the log is taken.

To go from the NPS= mode back to using only the density points in the table, give a command TABPT. The initial default for NPS= and TABPT is TABPT. Susequent defaults are to the last used procedure. If no $T=$ command is given and one is in the TABPT mode, only table temperatures and densities are used, and no interpolations are done for $p$ and $e$. Interpolations must always be done to obtain pdt, pdr, edt, and edr.

If you want other than a table temperature, type in the temperature in degrees kelvin. More than one $T=$ can be given. If $T=$ is given, no table temperatures are used. If no $T=$ 's are given, the temperatures are taken from the table. If $T=$ 's are given, interpolations are done.

If table temperatures are being used, NSTEP enables you to pick the interval used to space through the table. NSTEP=1 takes all table values, NSTEP $=2$ takes every second value, etc. One is the initial default. Subsequent defaults are to the last used value. 
RL $=$ REAL NUMBER

RU $=$ REAL NUMBER

$T L=$ REAL NUMBER

TU $=$ REAL NUMBER

$\mathrm{YL}=$ REAL NUMBER

$Y U=R E A L ~ N U M B E R$

$X L=R E A L ~ N U M B E R$

$X U=R E A L ~ N U M B E R$

3. Isochore Commands

RNPS $=$ INTEGER

RTABPT
It is possible to set a window on both density (lower density, RL, and upper density, RU) and temperature (lower temperature, TL, and upper temperature, TU). The initial defaults are to the table maxima and minima. Subsequent defaults are to the last used values. An exception to this arises if any last used values fall outside the table ranges. Then those window limits are set to their respective table limits. If you attempt to set a limit outside of a table's range, the code resets the limit to the table limit. Units are megagrams per meter cubed for densities and degrees kelvin for temperatures. If you are doing a graph with a log $\mathrm{x}$-axis and $\mathrm{RL}$ is less than or equal to $0.0, \mathrm{RL}$ is reset to $1.0 \mathrm{e}-10$.

It is possible to set a window on the y-variable (lower limit, $\mathrm{YL}$, and upper limit, YU). The same bounds apply to all y-variables even though the units are not the same. The initial defaults are to $\mathrm{YL}=1 . \mathrm{e}-3$ and $\mathrm{YU}=1 . \mathrm{e} 19$. Subsequent defaults are to the last used values. If the $y$-axis is $\log , Y L$ is set to $1 . e-3$ if $Y L$ is less than or equal to 0 . If the $y$-axis is $\log$, a check is made to see if YU is greater than 1.e22*YL or less than YL. If it is greater than 1.e22*YL or less than $\mathrm{YL}, \mathrm{YU}$ is set to 1.e22*YL. These checks are done whether the routine is defaulting to previous values or the user is typing in new values. The units are those appropriate for the $y$-axis variable.

These limits specify a window on the variable being plotted on the $\mathrm{x}$-axis. The discussion is the same as just given for the $\mathrm{y}$-axis window.

The integer gives the number of temperature points to be used for a curve. When the RNPS= command is given, interpolations are done to obtain the curve points. The temperature points are equally spaced between RTL and RTU. If the x-axis is log, the temperature points are equally spaced after the $\log$ is taken.

To go from the RNPS= mode back to using only the temperature points in the table, give a command RTABPT. The initial default for RNPS= and RTABPT is RTABPT. Subsequent defaults are to the last used procedure. If no $R=$ command is given and you are in the RTABPT mode, only table densities and temperatures are used and no interpolations are done for $\mathrm{p}$ and $\mathrm{e}$. Interpolations must always be done to obtain pdt, pdr, edt, and edr. 
$R=$ REAL NUMBER

RNSTEP $=$ INTEGER

RRL $=$ REAL NUMBER

RRU $=$ REAL NUMBER

RTL $=$ REAL NUMBER

RTU $=$ REAL NUMBER

RYL $=$ REAL NUMBER

RYU $=$ REAL NUMBER

RXL $=$ REAL NUMBER RXU $=$ REAL NUMBER
If you want other than a table density, type in the density in megagrams per meter cubed. More than one $R=$ can be given. If $R=$ is given, no table densities are used. If no $R=$ 's are given, the densities are taken from the table. If $R=$ 's are given, interpolations are done.

If table temperatures are being used, RNSTEP enables you to pick the interval used to space through the table. RNSTEP $=1$ takes all table values, $R$ NSTEP $=2$ takes every second, etc. One is the initial default. Subsequent defaults are to the last used value.

It is possible to set a window on both the density (lower density, RRL, and upper density, RRU) and temperature (lower temperature, RTL, and upper temperature, RTU). The initial defaults are to the table maxima and minima. Subsequent defaults are to the last used values. An exception to this arises if any last used values fall outside the table ranges. Then those window limits are set to their respective table limits. If you attempt to set a limit outside of a table's range, the code resets the limit to the table limit. Units are megagrams per meter cubed for densities and degrees kelvin for temperatures. If you are doing a graph with a $\log x$-axis and RTL is less than or equal '? 0.0, RTL is reset to $1.0 \mathrm{e}-10$.

It is possible to set a window on the y-variable (lower limit, RYL, and upper limit, RYU). The same bounds apply to all y-variables even though the units are not the same. The initial defaults are to $R Y L=1 . e-3$ and $R Y U=1 . e 19$. Subsequent defaults are to the last used values. If the $y$-axis is $\log , R Y L$ is set to $1 . e-3$ if RYL is less than or equal to 0 . If the $y$-axis is $\log$, a check is made to see if RYU is greater than 1.e22*RYL or less than RYL. If it is greater than 1.e22*RYL or less than RYL, RYU is set to 1.e22*RYL. These checks are done whether the routine is defaulting to previous values or the user is typing in new values. The units are those appropriate for the $y$-axis variable.

These limits specify a window on the variable being plotted on the $x$-axis. The discussion is the same as just given for the $y$-axis window. 
4. Hugoniot Commands

HNPS $=$ INTEGER

HTABPT

YCONS

NCONS

HRHO0 $=$ REAL NUMBER

HE0 $=$ REAL NUMBER

HP0 $=$ REAL NUMBER

$H R L=R E A L$ NUMBER

HRU $=$ REAL NUMBER
The integer gives the number of density points to be used for a curve. The density points are equally spaced between HRL and HRU. If the $x$-axis is log, the density points are equally spaced after the $\log$ of rho is taken.

To go from the HNPS= mode back to using only the density points in the table, give a command HTABPT. The initial default for HNPS $=$ and HTABPT is HTABPT. Subsequent defaults are to the last used procedure.

To determine a Hugoniot, you must specify initial shock conditions denoted by HRHOO, HEO, and HPO for initial density, initial internal energy, and initial pressure, respectively. If you impose thermodynamic consistency among these variables, you need only input two variables, in particular, $\mathrm{HRHOO}$ and HEO for this code. However, for some uses (foams), all three variables, HRHOO. HEO, and HPO, are needed without thermodynamic consistency. Use YCONS and NCONS to switch between the two modes. YCONS is the initial default and means that HPO is to be determined from HRHOO and HEO in a thermodynamically consistent way. NCONS means that HRHOO, HEO, and HPO are independent of each other. Defaults other than the initial are to whatever was last used.

These commands give the initial parameters for the Hugoniot. HRHOO is the initial density in megagrams per meter cubed, HEO is the initial internal energy in megajoules per kilogram, and HPO is the initial pressure in gigapascals. More than one group of such commands can be given. The code takes the commands in order. The initial defaults are $\mathrm{HRHOO}=$ rhoO (from the SESAME table), $H E 0=0.0$, and $H P O=0.0$. Subsequent defaults are is last used values. HRHOO cannot be set outside the bounds of the table and will be reset to the lower table bound if it is outside. If YCONS is on, HPO is always calculated from $\mathrm{HR} \mathrm{HOO}$ and $\mathrm{HEO}$, and any $\mathrm{HPO}=$ command is ignored.

It is possible to set a window on the density (lower density, HRL, and upper density, HRU). The initial defaults are HRL= ThoO and HRU $=\min \left(2.5^{*} \mathrm{RHOO}\right.$, upper table limit). (rhoO is from the SESAME table.) Subsequent defaults are to the last used values. Note that HRL is set to HRHOO if HRL is less than HRHOO, and HRU is set to the table maximum if HRU is greater than the table maximum. Units are megagrams per meter cubed. 
HYL=REAL NUMBER

HYU $=$ REAL NUMBER

HXL $=$ REAL NUMBER HXU $=$ REAL NUMBER

HERR

HNERR

5. Adiabat Commands

SNPS $=$ INTEGER

STABPT

SRHOO $=$ REAL NUMBER SE0 $=$ REAL NUMBER
It is possible to set a window on the y-variable (lower limit, HYL, and upper limit, HYU). The same bounds apply to all $y$-variables even though the units are not the same. The initial defaults are to $H Y L=1 . e-3$ and $H Y U=1 . e 19$. Subsequent defaults are to the last used values. If the $y$-axis is $\log , H Y L$ is set to $1 . e-3$ if HYL is less than or equal to 0 . If the $y$-axis is $\log$, a check is made to see if HYL is greater than $1 . e 22^{*} \mathrm{HYL}$ or less than HYL. If it is greater than 1.e22*HYL or less than HYL, HYU is set to $1 . e 22^{*} \mathrm{HYL}$. These checks are done whether the routine is defaulting to previous values or the user is typing in new values. The units are those appropriate for the $y$-axis variable.

These limits specify a windoi' on the variable being plotted on the $x$-axis. The discussion is the same as just given for the $y$-axis window.

The routine that calculates Hugoniot parameters can have difficulties, in particular, at rho=HRHOO and for large rho. A message is printed if the routine is having difficulty. If you want to know the problem densities, give the command HERR. The problem densities will be printed if you repeat the calculation. HNERR turns off the printing. The initial default is HNERR. Subsequent defaults are to what was last done.

The integer gives the number of density points to be used for a curve. The density points are equally spaced between SRL and SRU. If the $x$-axis is $\log$, the density points are equally spaced after the $\log$ of rho is taken.

To go from the SNPS = mode back to using only the density points in the table, give a command STABPT. The initial default for SNPS= and STABPT is STABPT. Subsequent defaults are to the last used procedure.

These commands select the desired adiabat. The adiabat drawn will go through SRHOO and SEO, where SRHOO is a density in megagrams per meter cubed and SE0 is an energy in megajoules per kilogram. More than one group of such commands can be given. The code takes the commands in order. The initial defaults are SHROO= rhoO (from the SESAME tialle; and $\mathrm{SEO}=0.0$. Subsequent defaults are to last used values. SRHOO cannot be set outside the bounds of the table and will be reset to 
$S R L=R E A L ~ N U M B E R$

SRU $=$ REAL NUMBER

SYL=REAL NUMBER

$S Y U=R E A L$ NUMBER

$S X L=R E A L N U M B E R$

$S X U=R E A L$ NUMBER

SERR

SNERR the lower table bound if it is outside. There is no freedom to select SPO as in the Hugoniot mode.

It is possible to set a window on the density (lower density, SRL, and upper density, SRU). The initial defaults are $S R L=$ rho0 and $S R U=\min \left(2.5^{*}\right.$ rho0, upper table limit). (rho0 is from the SESAME table.) Subsequent defaults are to the last used values. Note that SRL is set to the lower table limit if SRL is less than the lower table limit, and SRU is set to the table maximum if SRU is greater than the table maximum. Units are megagrams per meter cubed.

It is possible to set a window on the $y$-variable (lower limit, SYL, and upper limit, SYU). The same bounds apply to all y-variables even though the units are not the same. The initiai defaults are to $S Y L=1 . e-3$ and $S Y U=1 . e 19$. Subsequent defaults are to the last used values. If the $y$-axis is $\log$, SYL is set to $1 . e-3$ if SYL is less than or equal to 0 . If the $y$-axis is $\log$, a check is made to see if SYU is greater than 1.e22*SYL or less than SYL. If it is greater than $1 . e 22^{*}$ SYL or less than SYL, SYU is set to $1 . \mathrm{e} 22^{*} \mathrm{SYL}$. These checks are done whether the routine is defaulting to previous values or the user is typing in new values. The units are those appropriate for the $y$-axis variable.

These limits specify a window on the variable being plotted on the $x$-axis. The discussion is the same as just given for the $y$-axis window.

The routine that calculates adiabat parameters can have difficulties. A message is printed if there is a problem. If you want to know the problem densities, give the command SERR. The problem densities will be printed if you repeat the calculation. SNERR turns off the printing. The initial default is SNERR. Subsequent defaults are to what was last done.

\section{EXAMPLES}

Figures $I$ and 2 show a sample run with a number of graphs. The first page has the initial input for the run. This input (material number and device number) is the minimum amount of information needed to construct a graph. All other parameters are defaulted. In the following pages, the commands given after CHANGES? on a graph are the input that produces the next graph. For this run, we are working with a public file SESAME and a local file SES for the equation of state libraries.

A few words describing the graphs are in order. All is obvious except the table on the left side of the graphs. The table headings contain the material numbers of materials being presented; contractions of the words isotherm, isochore, Hugoniot, or adiabat describe the types of curves shown. The 1 or 2 following 
the contracted descriptor denotes SESAME1 or SESAME2 format. Under the headings are labels for the individual curves being plotted. For isotherms these are temperatures; isochores will have densitites; Hugoniots will list HEO; and adiabats will list SEO. The user has to retain HRHOO, SRHOO, and HPO; they are not listed on the graphs. 


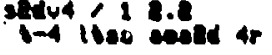

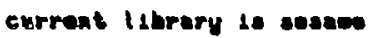

1npat dete

Fig. 1. Initial input to construct a graph. 


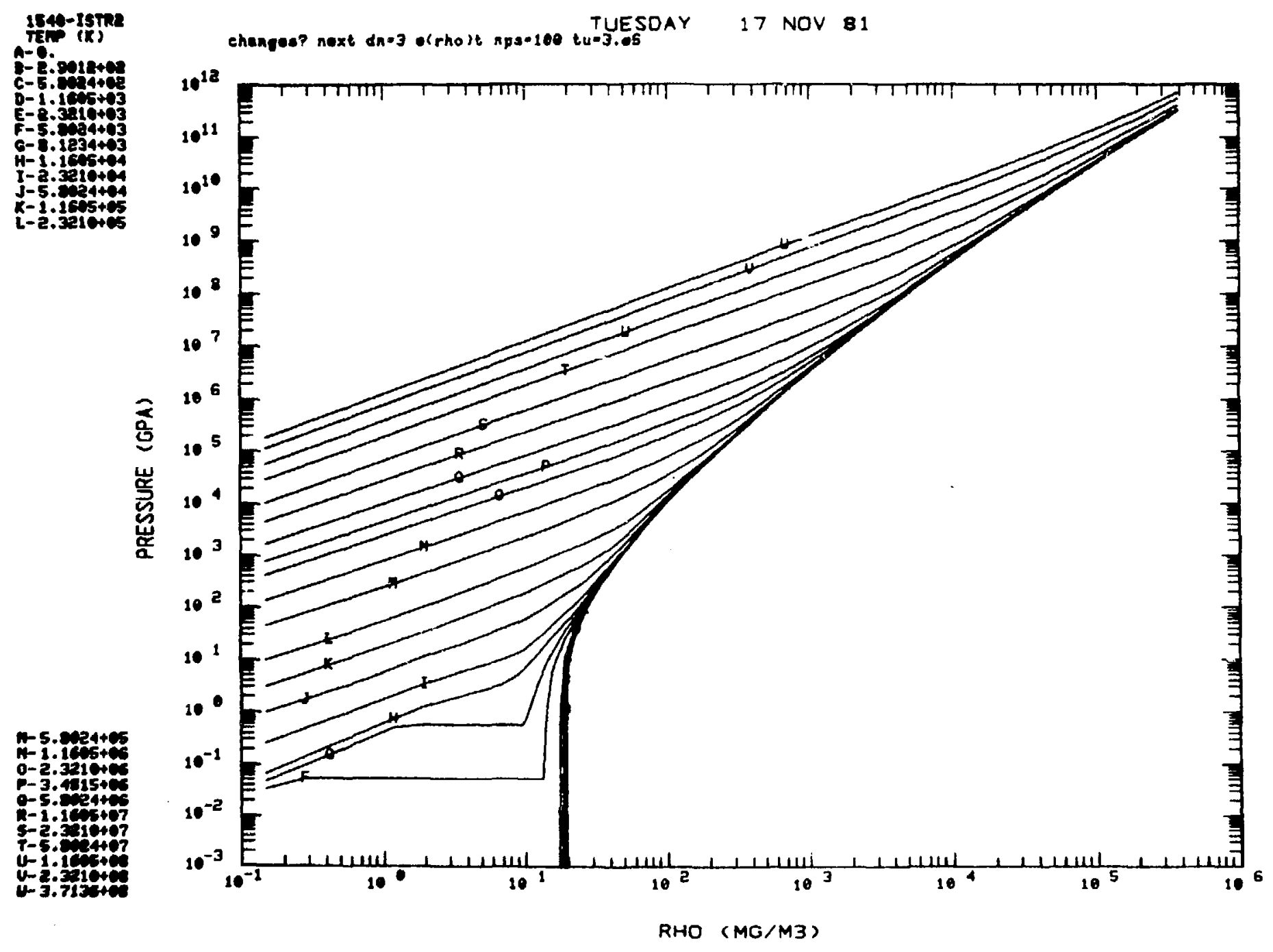

T-4 LTSS SESED AR 


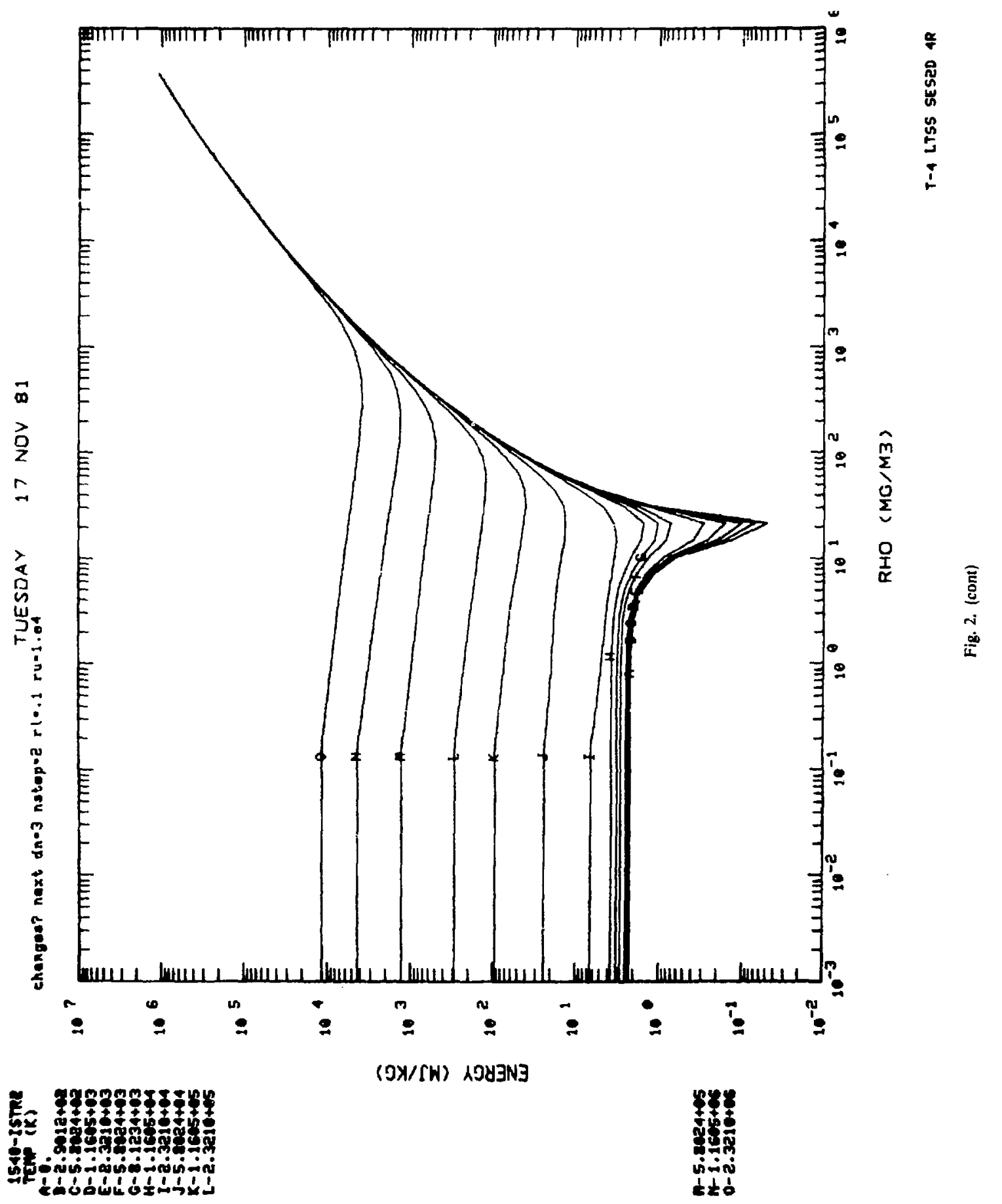



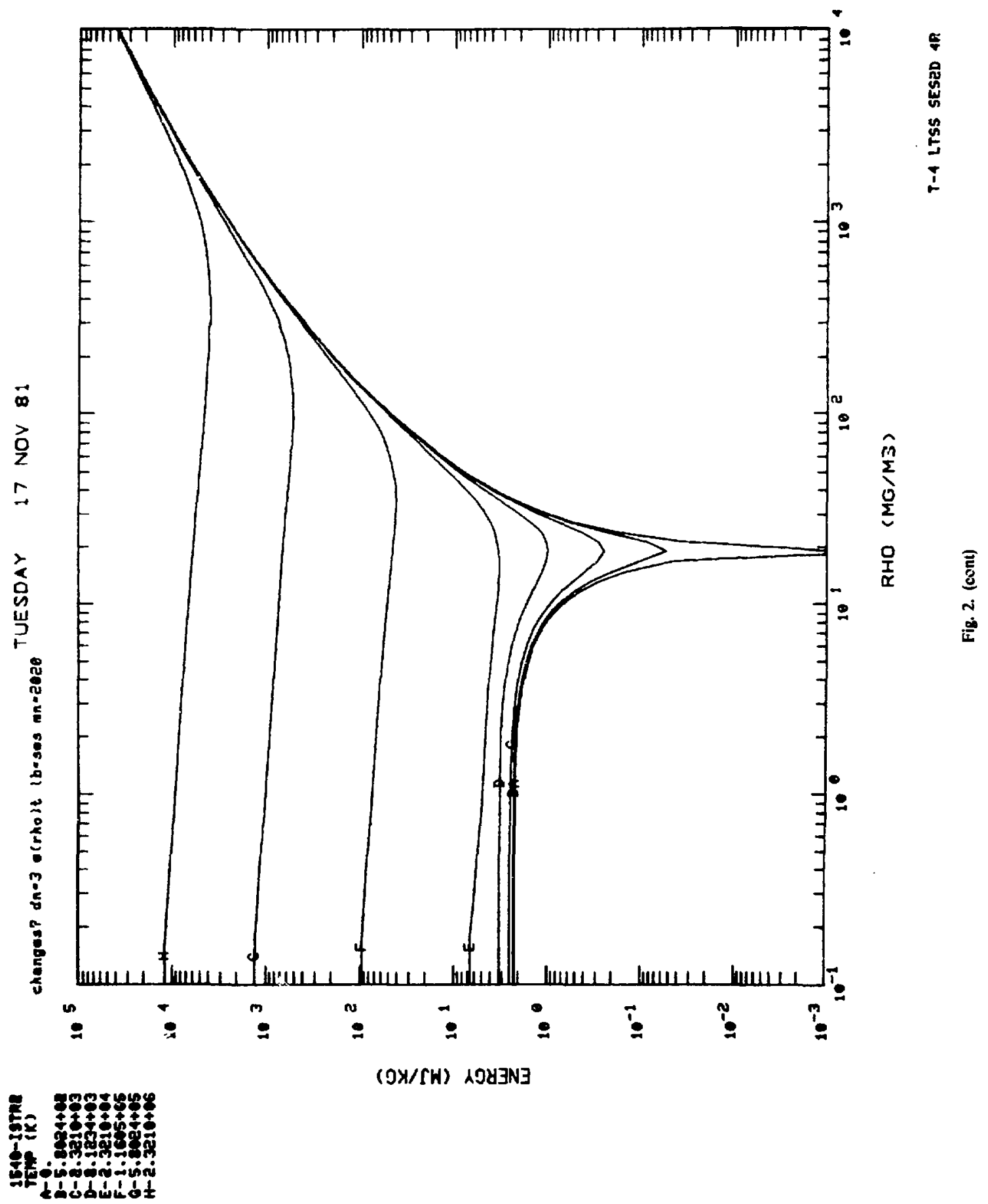

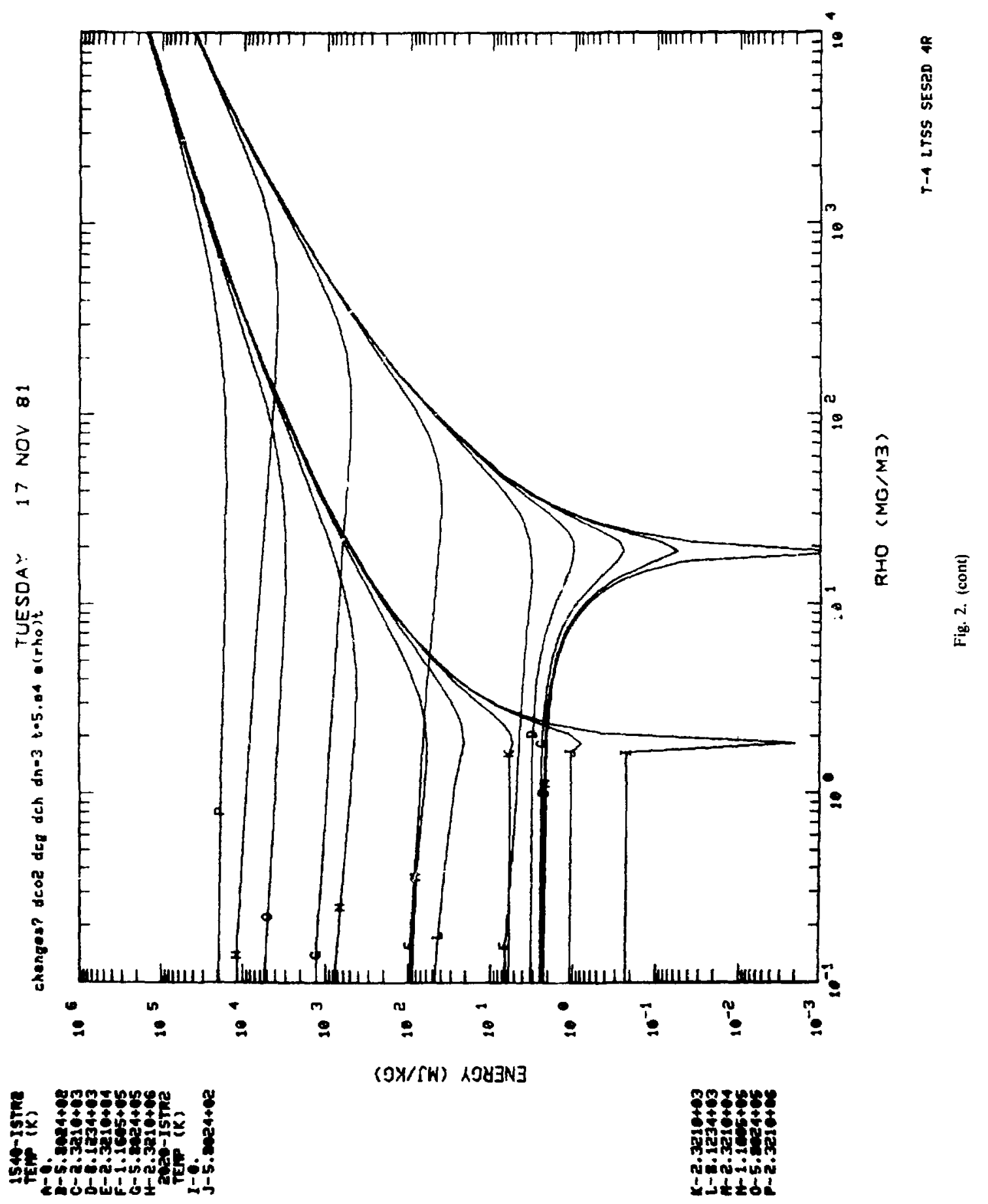


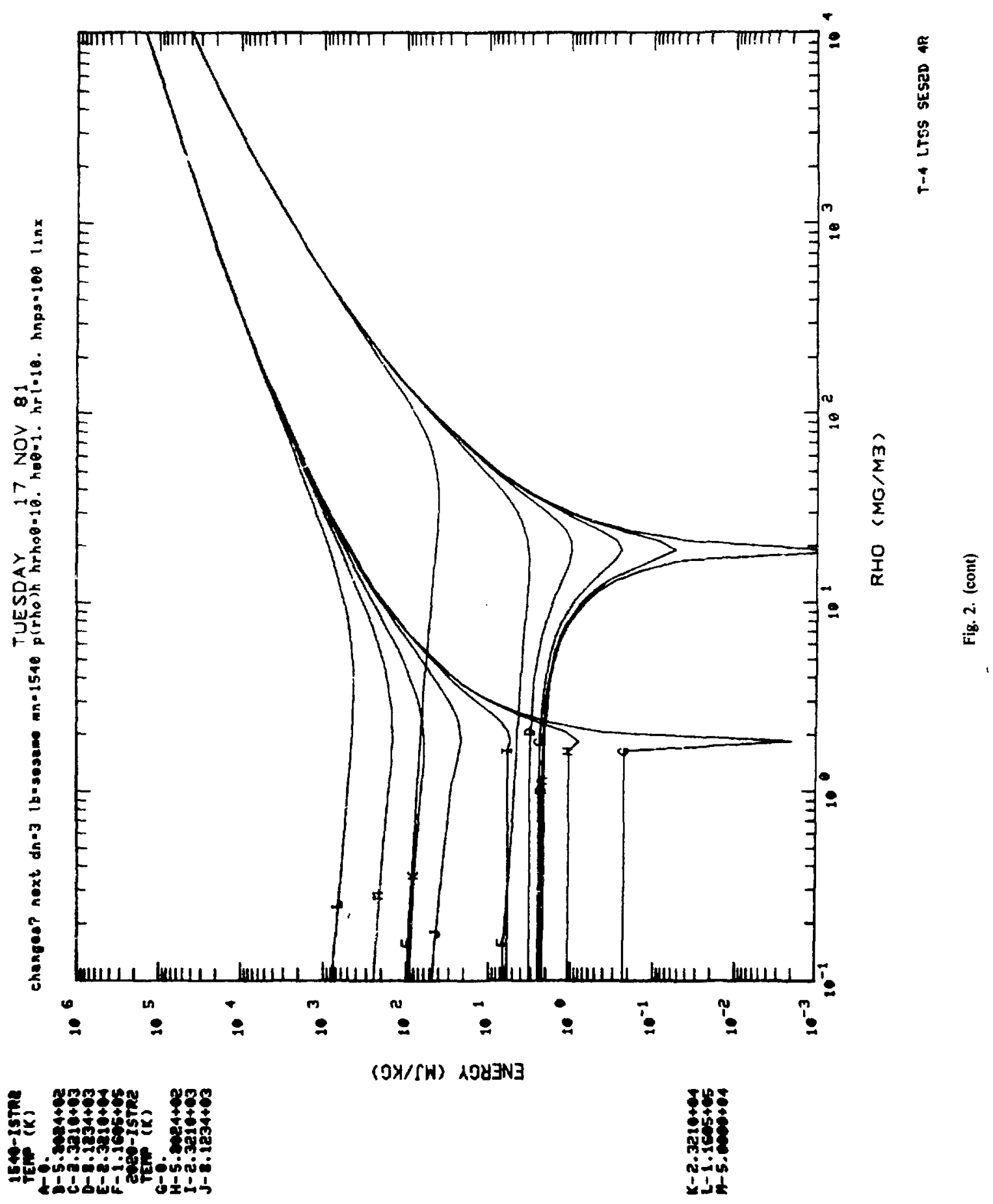




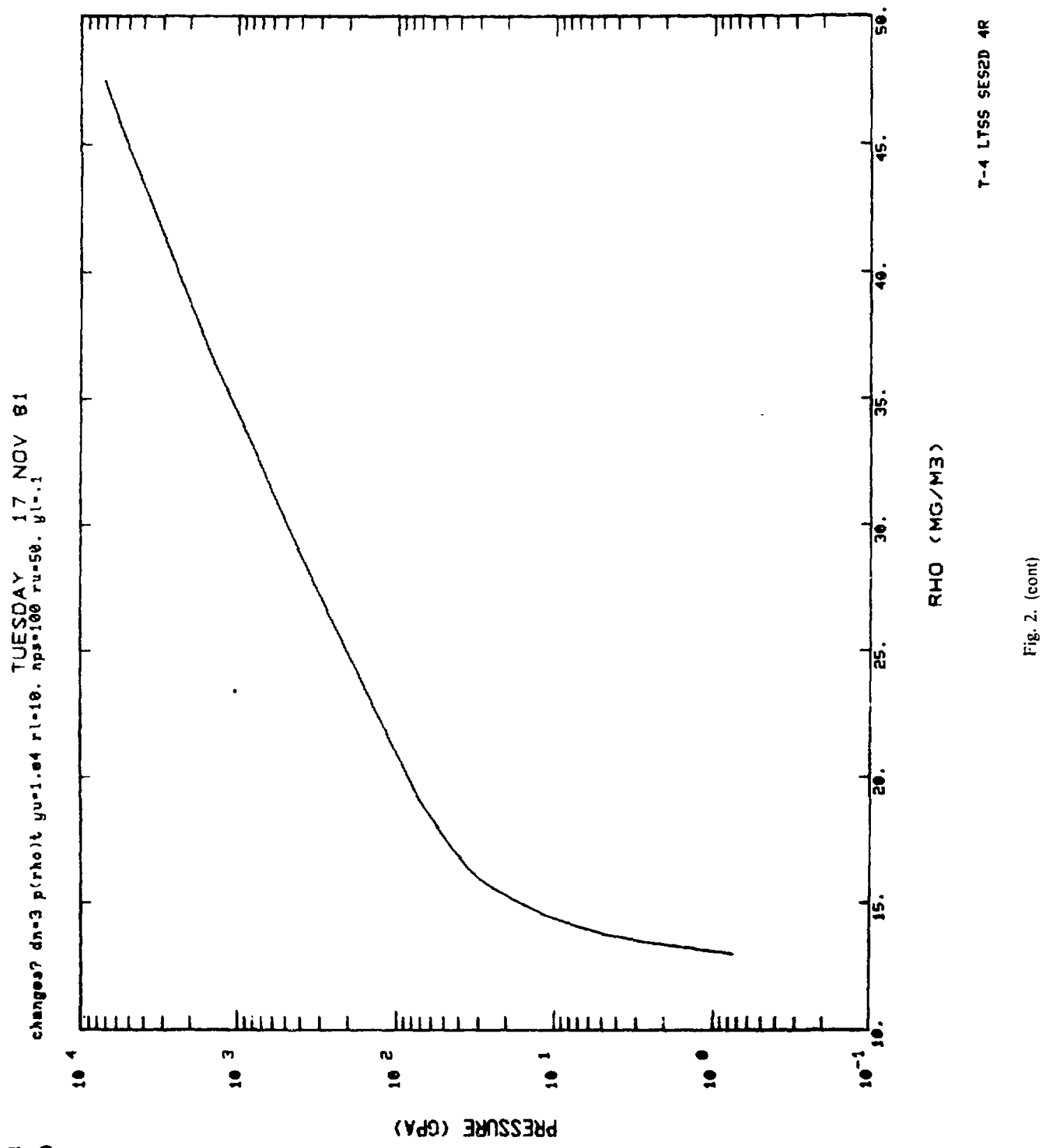



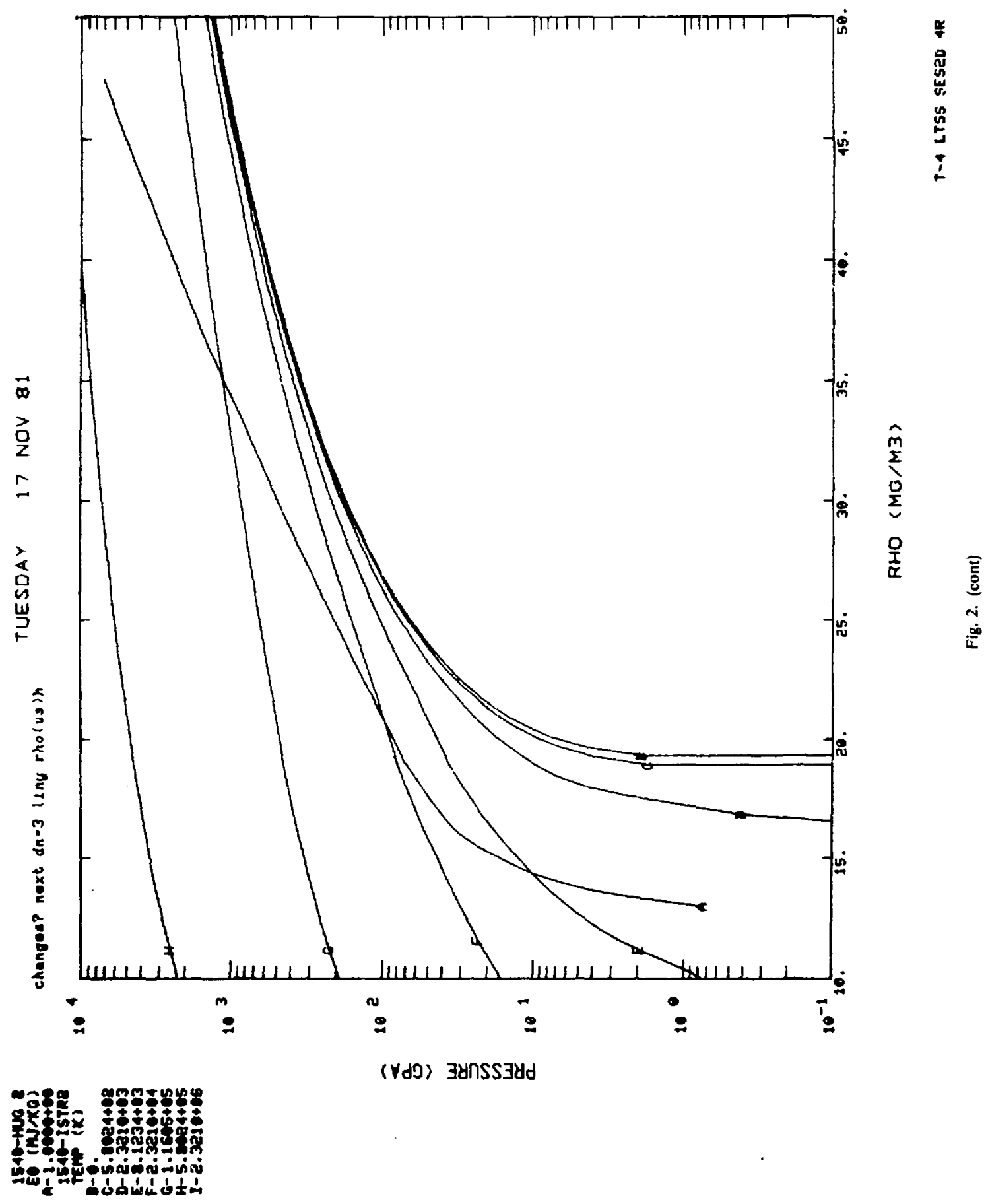


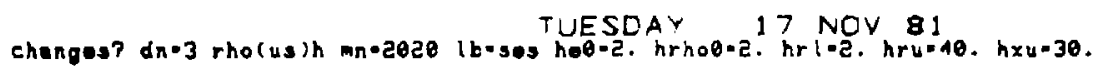

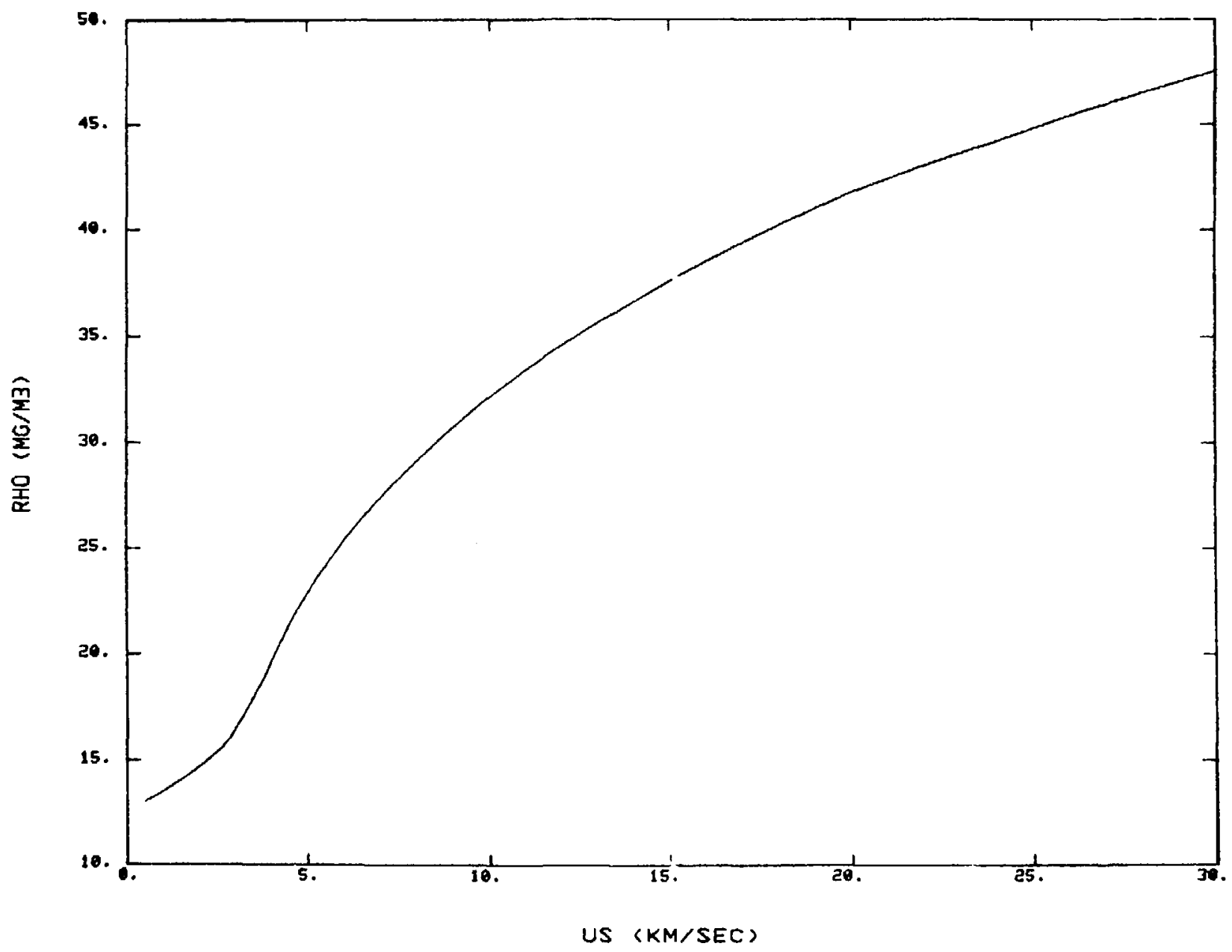




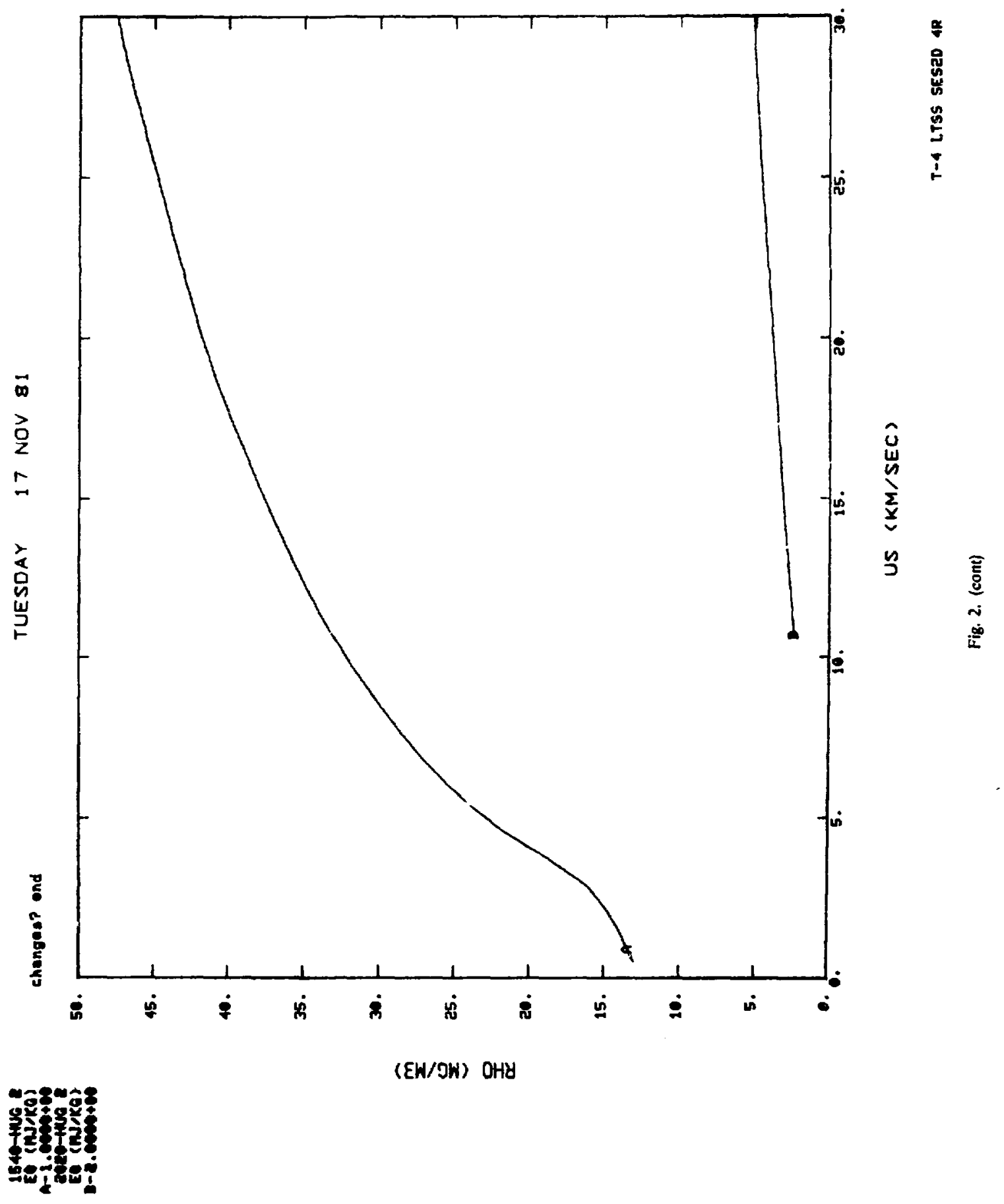

